\title{
Genome-wide analysis of the WRKY gene family in the cucumber genome and transcriptome-wide identification of WRKY transcription factors that respond to biotic and abiotic stresses
}

\author{
Chunhua Chen ${ }^{1,2^{*}} \mathbb{D}$, Xueqian Chen ${ }^{1,2}$, Jing Han ${ }^{1,2}$, Wenli Lu ${ }^{1,2}$ and Zhonghai Ren ${ }^{1,2^{*}}$
}

\begin{abstract}
Background: Cucumber (Cucumis sativus L.) is an economically important vegetable crop species. However, it is susceptible to various abiotic and biotic stresses. WRKY transcription factors play important roles in plant growth and development, particularly in the plant response to biotic and abiotic stresses. However, little is known about the expression pattern of WRKY genes under different stresses in cucumber.

Results: In the present study, an analysis of the new assembly of the cucumber genome (v3.0) allowed the identification of 61 cucumber WRKY genes. Phylogenetic and synteny analyses were performed using related species to investigate the evolution of the cucumber WRKY genes. The 61 CsWRKYs were classified into three main groups, within which the gene structure and motif compositions were conserved. Tissue expression profiles of the WRKY genes demonstrated that 24 CSWRKY genes showed constitutive expression (FPKM $>1$ in all samples), and some WRKY genes showed organ-specific expression, suggesting that these WRKYS might be important for plant growth and organ development in cucumber. Importantly, analysis of the CsWRKY gene expression patterns revealed that five CSWRKY genes strongly responded to both salt and heat stresses, 12 genes were observed to be expressed in response to infection from downy mildew and powdery mildew, and three CsWRKY genes simultaneously responded to all treatments analysed. Some CSWRKY genes were observed to be induced/repressed at different times after abiotic or biotic stress treatment, demonstrating that cucumber WRKY genes might play different roles during different stress responses and that their expression patterns vary in response to stresses.

Conclusions: Sixty-one WRKY genes were identified in cucumber, and insight into their classification, evolution, and expression patterns was gained in this study. Responses to different abiotic and biotic stresses in cucumber were also investigated. Our results provide a better understanding of the function of CSWRKY genes in improving abiotic and biotic stress resistance in cucumber.
\end{abstract}

Keywords: Cucumber, WRKY, Abiotic stress, Biotic stress, Expression profiling

\footnotetext{
* Correspondence: chenchunhua@sdau.edu.cn; zhren@sdau.edu.cn

${ }^{1}$ State Key Laboratory of Crop Biology, Shandong Collaborative Innovation

Center of Fruit \& Vegetable Quality and Efficient Production, Tai'an, People's Republic of China

Full list of author information is available at the end of the article
}

C C The Author(s). 2020 Open Access This article is licensed under a Creative Commons Attribution 4.0 International License, which permits use, sharing, adaptation, distribution and reproduction in any medium or format, as long as you give appropriate credit to the original author(s) and the source, provide a link to the Creative Commons licence, and indicate if changes were made. The images or other third party material in this article are included in the article's Creative Commons licence, unless indicated otherwise in a credit line to the material. If material is not included in the article's Creative Commons licence and your intended use is not permitted by statutory regulation or exceeds the permitted use, you will need to obtain permission directly from the copyright holder. To view a copy of this licence, visit http://creativecommons.org/licenses/by/4.0/ The Creative Commons Public Domain Dedication waiver (http://creativecommons.org/publicdomain/zero/1.0/) applies to the data made available in this article, unless otherwise stated in a credit line to the data. 


\section{Background}

Throughout their lifecycle, plants frequently encounter many different types of stresses that severely prevent them from reaching optimal growth and that may have a great impact on yield production [1-4]. Some of these stresses are abiotic stress factors such as temperature, drought, salt, and heavy metal stress. By contrast, biotic stress factors involve the interaction of plants with insect, nematode, viral, bacterial, fungal, or oomycete origins that use the plant as a food source $[2,5]$. To withstand or cope with these different stresses, plants have evolved a series of adjustment mechanisms including a broad regulation of numerous genes to mediate plant physiological and biochemical processes $[6,7]$. Therefore, the study of genes involved in these mechanisms is important for the development of biotechnological tools to enhance desirable agronomic traits, such as plant growth and productivity. Transcription factors (TFs), including members of the AP2/ERF, NAC, MYB, and WRKY families, participate in plant tolerance against abiotic and biotic stresses by modulating the expression of defence-related genes [8-14].

The WRKY gene family is one of the largest and most extensively studied TF families in higher plants [15]. Since the first WRKY gene was cloned in sweet potato, the identification of WRKY genes has been performed in various plant species, including Arabidopsis thaliana (72) [16], Oryza sativa (103) [17], Zea mays (120) [18], and Solanum lycopersicum (81) [19]. WRKY TFs share a conserved DNA-binding domain that contains a highly conserved WRKYGQK heptapeptide followed by a $\mathrm{C} 2 \mathrm{H} 2$ - or $\mathrm{C} 2 \mathrm{HC}$ type zinc finger motif $[15,20]$. WRKY TFs function by recognizing and binding W-box cis-elements (TTGACC/ $\mathrm{T}$ ) of target genes, and both the heptapetide sequence and zinc finger motif are required for this high binding activity $[15,21,22]$. Based on the number of WRKY domains and the type of zinc fingers, WRKY TFs can be classified into three phylogenetically distinct groups: Group I WRKYs, which have two WRKY domains; Group II WRKYs, which have one WRKY domain, while both group I and II WRKYs contain one $\mathrm{C} 2 \mathrm{H} 2$-type zinc finger motif (C-X45-C-X22-23-H-X1-H); and Group III members, which feature one WRKY domain and a C2HC-type motif (C$\mathrm{X} 7-\mathrm{C}-\mathrm{X} 23-\mathrm{H}-\mathrm{X} 1-\mathrm{C})$. Moreover, Group II is further divided into five subgroups (IIa-IIe) based on phylogenetic analyses [23-25].

WRKY TFs have been reported to be involved in many aspects of plant development $[25,26]$, including senescence [27, 28], trichome development [29], biosynthesis of secondary metabolites [21, 30-32], flowering [33, 34], and seed development and germination [35-37]. Substantial evidence has demonstrated that many WRKY genes also participate in various stress responses. For example, the expression of 18 WRKY genes was shown to be induced by exposure to salt stress in the roots of Arabidopsis [38]. WRKY6 and WRKY42 were identified to participate in the response to low Pi stress by regulating PHO1 expression [39]. WRKY TFs from Arabidopsis were also shown to regulate the defence response positively and/or negatively against bacterial pathogens [16, 40], fungal pathogens [41-43] and nematodes [44, 45]. The expression levels of 13 OsWRKY genes from rice were examined in response to different treatments, including salt, polyethylene glycol (PEG), and cold or heat stresses, and 10 WRKY genes were down- or upregulated in response to these abiotic stresses. Moreover, WRKY proteins from tomato (S. lycopersicum) [19, 46], Brassica napus [47], soybean (Glycine max) [48], rice (O. sativa) [49, 50], wheat (Triticum aestivum L.) [51] and other plant species were shown to play critical roles in the response to various biotic and abiotic stresses.

According to the above mentioned discussion, WRKY TFs may participate in multiple pathways, leading to an array of physiological responses. The elucidation of the evolution and duplicative expansion of WRKY genes seems to be related to the diversity of their functions $[20,24]$. The evolutionary studies of the WRKY gene family and large-scale genome-wide analyses of WRKY genes indicated that Group IIa genes, which compose the group with the fewest number of members, were the last to evolve and appear to have originated from Group IIb genes. Furthermore, Group IIa TFs play many important roles in the regulation of biotic and abiotic stress responses [20].

Cucumber (Cucumis sativus L.), one of the most economically important vegetable crop species, produces tender fruits that are edible organs [52, 53]. In addition, cucumber is extensively used as a model system in the study of sex determination, vascular biology, and induced defence responses [54]. In cucumber cultivation, yield and quality are frequently affected by different types of biotic and abiotic stresses, leading to a decline in cucumber output. Therefore, the identification of new functional genes for resistance to stresses is gaining considerable interest. Based on the cucumber genome (v1.0), 57 WRKY genes were identified, and 23 of them had been shown to be differentially expressed in response to at least one abiotic stress [55]. Low-coverage Sanger sequences and short high-coverage Illumina sequences were used to assemble draft cucumber genomes (v1.0 and v2.0); thus, these genomes are incomplete and of low quality. A high-quality and complete cucumber genome assembly (v3.0) is currently available for use in comparative genomics and genetic research [56]. Here, a new genome-wide identification of cucumber WRKYs was performed by the use of the cucumber (Chinese Long, 9930) genome (v3.0). We identified 61 WRKY genes and classified them into three groups. 
Comprehensive analyses including the gene structures, chromosomal locations, conserved protein domains, and phylogenetic analysis were further performed. The expression profiles of genome-wide CsWRKY genes in cucumber plants under different stresses were investigated. Our results will provide valuable clues for future work on the function of WRKYs in cucumber.

\section{Results}

\section{The cucumber genome contains 61 WRKY genes}

In a previous study, 57 WRKY genes were identified in the cucumber (Chinese long, 9930) genome (v1.0) [55]. Recently, an updated version (v3.0) was released in CuGenDB (http://cucurbitgenomics.org/), and the v1.0 was eliminated. Therefore, we identified cucumber WRKY genes in the cucumber genome (v3.0), and 61 WRKY genes were identified by a hidden Markov model (HMM) search using the WRKY domain (PF03106). These genes were proven to contain WRKY domains according to Pfam and SMART analysis. Among the previous 57 WRKY genes, five (CsWRKY53-CsWRKY57) were not conclusively mapped to any chromosome on the basis of the cucumber genome (v1.0) [55]; however, all 61 of the WRKY genes identified in this study could be mapped onto the chromosomes on the basis of the current version of the cucumber genome (v3.0) (Additional file 1: Fig. S1). There are seven chromosomes in the cucumber genome; the WRKY genes were not evenly dispersed across all chromosomes. Chromosome 3 harboured the highest number of CsWRKY genes (15, $24.59 \%)$, while only five $(8.20 \%)$ were found on chromosome 5. Except for chromosomes 1 and 4, the number of WRKY genes we identified mapped onto every chromosome was at least one more than that in a previous study (Additional file 2: Fig. S2). Based on their order on the chromosomes, the WRKY genes identified in this study were renamed CsWRKY1 to CsWRKY61 (Additional file 1: Fig. S1), and this nomenclature approach was identical to that used in the previous study. A comparison of the currently known WRKY TFs in the cucumber genomes (Gy14, v1.0; 9930, v2.0 and v3.0) is listed in Additional file 3: Table S1.

For these 61 WRKY genes, the length of the coding DNA sequence (CDS) and the protein sequence, the protein molecular weight (MW), and the isoelectric point (pI) were analysed (Table 1 and Additional file 3: Table S1). The largest protein was CsWRKY8, comprising 1118 amino acids (aa), whereas the smallest one was CsWRKY47 (119 aa), corresponding to MWs ranging from 13.95 (CsWRKY47) to 124.59 (CsWRKY8) kDa. The pIs of the WKRYs ranged from 5.11 (CsWRKY10) to 10.08 (CsWRKY54). According to the predicted results of subcellular localization, all these CsWRKY proteins might be localized to the nucleus. The subcellular localization of CsWRKY50 (in this paper named CsWRKY47) [53] could support this claim.

\section{Multiple sequence alignment, phylogenetic relationship, and classification of CsWRKY proteins}

The WRKY domains, which comprise approximately 60 aa, of the newly identified CsWRKYs were first aligned, and seven AtWRKY domains (AtWRKY58, 56, 21, 35, 46,40 , and 6) from each group or subgroup were randomly selected as representatives for analysis. The highly conserved sequence WRKYGQK was found within a total of 58 CsWRKY proteins, while the others (CsWRKY10, CsWRKY47, and CsWRKY50) had a single amino acid substitution: K for Q (Fig. 1 and Table 1).

A phylogenetic tree was constructed using the neighbour-joining (NJ) method by MEGA 5.0 software with 1000 bootstrap tests and based on multiple alignments of cucumber and Arabidopsis [16] WRKY domain aa sequences (Additional file 4: Table S2). As shown in Fig. 2, cucumber WRKY proteins could be categorized into three large groups (Group I-III) on the basis of the classifications of WRKYs in Arabidopsis [25]. Among the sequences of the 61 CsWRKY proteins, 11 sequences were assigned to Group I, 43 sequences belonged to Group II, and seven were assigned to Group III. In Group I, 10 members contained two WRKY domains (an N-terminal and a C-terminal WRKY domain), whereas CsWRKY42 had lost its C-terminal WRKY GQK-like stretch; these 11 members all harboured $\mathrm{C} 2 \mathrm{H} 2$-type zinc finger motifs (C-X4-C-X22-24-H-X-H). The members of Group II contained a WRKY domain and could be further classified into five subgroups (IIaIIe). Moreover, three members were classed in IIa, which was the group with the smallest number of members; 5 , IIb; 20, IIc; 7, IId; and 8, IIe. Although most of the members of Group II had integral C2H2-type zinc finger motifs, partial absence of the zinc finger motif sequence was present in CsWRKY14 and CsWRKY47. Except for CsWRKY40, whose zinc finger motif was almost entirely absent, the CsWRKYs classed in Group III harboured a WRKY domain and contained a C2HC-type zinc finger motif. The 'leucine-rich repeat' (LRR) motif, which is a typical domain of resistance (R) proteins and is found in WRKY proteins of some species, such as Arabidopsis and rice, was not observed in the WRKY proteins of cucumber.

The Group IIa WRKY genes were found to be the last to evolve, as these genes compose the only group absent from the spike moss Selaginella moellendorffii [20]. The WRKY gene family members in many species have been identified, and their detailed numbers were listed in Table 2. Thus, we investigated the duplication and diversification of Group IIa WRKYs during evolution based on the available WRKY IIa genes in different species, 
Table 1 Features of WRKY genes identified in cucumber

\begin{tabular}{|c|c|c|c|c|c|c|c|c|}
\hline \multirow[t]{2}{*}{ Name } & \multirow[t]{2}{*}{ Gene ID (V3) } & \multirow[t]{2}{*}{ ORF } & \multirow{2}{*}{$\begin{array}{l}\text { Intron } \\
\text { number }\end{array}$} & \multirow[t]{2}{*}{ AA } & \multicolumn{3}{|l|}{ WRKY domain } & \multirow[t]{2}{*}{ Group } \\
\hline & & & & & Conserved heptapeptide & Zinc-finger type & $\begin{array}{l}\text { Domain } \\
\text { number }\end{array}$ & \\
\hline CsWRKY1 & CsaV3_1G002180 & 1773 & 4 & 590 & WRKYGQK & $\mathrm{C} 2 \mathrm{H} 2$ & 1 & II $\mathbf{b}$ \\
\hline CsWRKY2 & CsaV3_1G004720 & 1731 & 4 & 576 & WRKYGQK/WRKYGQK & $\mathrm{C} 2 \mathrm{H} 2$ & 2 & I \\
\hline CsWRKY3 & CsaV3_1G007870 & 1845 & 3 & 614 & WRKYGQK & $\mathrm{C} 2 \mathrm{H} 2$ & 1 & II $\mathbf{b}$ \\
\hline CsWRKY4 & CsaV3_1G028960 & 1521 & 4 & 506 & WRKYGQK/WRKYGQK & $\mathrm{C} 2 \mathrm{H} 2$ & 2 & I \\
\hline CsWRKY5 & CsaV3_1G032000 & 849 & 2 & 282 & WRKYGQK & $\mathrm{C} 2 \mathrm{H} 2$ & 1 & II d \\
\hline CsWRKY6 & CsaV3_1G033110 & 858 & 2 & 285 & WRKYGQK & $\mathrm{C} 2 \mathrm{H} 2$ & 1 & II e \\
\hline CsWRKY7 & CsaV3_1G037680 & 1242 & 2 & 413 & WRKYGQK & $\mathrm{C} 2 \mathrm{H} 2$ & 1 & II e \\
\hline CsWRKY8 & CsaV3_1G044520 & 3357 & 6 & 1,118 & WRKYGQK/WRKYGQK & $\mathrm{C} 2 \mathrm{H} 2$ & 2 & 1 \\
\hline CsWRKY9 & CsaV3_2G013650 & 1047 & 2 & 348 & WRKYGQK & $\mathrm{C} 2 \mathrm{H} 2$ & 1 & II d \\
\hline CsWRKY10 & CsaV3_2G017720 & 618 & 2 & 205 & WRKYGKK & $\mathrm{C} 2 \mathrm{H} 2$ & 1 & II c \\
\hline CsWRKY11 & CsaV3_2G017760 & 741 & 3 & 246 & WRKYGQK & $\mathrm{C} 2 \mathrm{H} 2$ & 1 & II c \\
\hline CsWRKY12 & CsaV3_2G032460 & 939 & 3 & 312 & WRKYGQK & $\mathrm{C} 2 \mathrm{H} 2$ & 1 & II a \\
\hline CsWRKY13 & CsaV3_2G032470 & 612 & 3 & 203 & WRKYGQK & $\mathrm{C} 2 \mathrm{H} 2$ & 1 & II a \\
\hline CsWRKY14 & CsaV3_2G034030 & 681 & 2 & 226 & WRKYGQK & $\mathrm{C} 2$ & 1 & II c \\
\hline CsWRKY15 & CsaV3_2G035630 & 882 & 2 & 293 & WRKYGQK & $\mathrm{C} 2 \mathrm{H} 2$ & 1 & II d \\
\hline CsWRKY16 & CsaV3_3G003840 & 2244 & 4 & 747 & WRKYGQK/WRKYGQK & $\mathrm{C} 2 \mathrm{H} 2$ & 2 & 1 \\
\hline CsWRKY17 & CsaV3_3G004410 & 1602 & 5 & 533 & WRKYGQK/WRKYGQK & $\mathrm{C} 2 \mathrm{H} 2$ & 2 & I \\
\hline CsWRKY18 & CsaV3_3G007160 & 660 & 3 & 219 & WRKYGQK & $\mathrm{C} 2 \mathrm{H} 2$ & 1 & II c \\
\hline CsWRKY19 & CsaV3_3G008170 & 1014 & 2 & 337 & WRKYGQK & $\mathrm{C} 2 \mathrm{HC}$ & 1 & III \\
\hline CsWRKY20 & CsaV3_3G008580 & 546 & 1 & 181 & WRKYGQK & $\mathrm{C} 2 \mathrm{H} 2$ & 1 & II c \\
\hline CsWRKY21 & CsaV3_3G008610 & 1011 & 2 & 336 & WRKYGQK & $\mathrm{C} 2 \mathrm{H} 2$ & 1 & II e \\
\hline CsWRKY22 & CsaV3_3G015290 & 972 & 2 & 323 & WRKYGQK & $\mathrm{C} 2 \mathrm{H} 2$ & 1 & II c \\
\hline CsWRKY23 & CsaV3_3G018790 & 1521 & 4 & 506 & WRKYGQK & $\mathrm{C} 2 \mathrm{H} 2$ & 1 & II b \\
\hline CsWRKY24 & CsaV3_3G021980 & 1101 & 2 & 366 & WRKYGQK & $\mathrm{C} 2 \mathrm{HC}$ & 1 & III \\
\hline CsWRKY25 & CsaV3_3G026600 & 999 & 3 & 332 & WRKYGQK & $\mathrm{C} 2 \mathrm{H} 2$ & 1 & II c \\
\hline CsWRKY26 & CsaV3_3G026920 & 513 & 1 & 170 & WRKYGQK & $\mathrm{C} 2 \mathrm{H} 2$ & 1 & II c \\
\hline CsWRKY27 & CsaV3_3G033000 & 990 & 1 & 329 & WRKYGQK & $\mathrm{C} 2 \mathrm{HC}$ & 1 & III \\
\hline CsWRKY28 & CsaV3_3G033350 & 1431 & 4 & 476 & WRKYGQK/WRKYGQK & $\mathrm{C} 2 \mathrm{H} 2$ & 2 & I \\
\hline CsWRKY29 & CsaV3_3G035430 & 1419 & 4 & 472 & WRKYGQK/WRKYGQK & $\mathrm{C} 2 \mathrm{H} 2$ & 2 & 1 \\
\hline CsWRKY30 & CsaV3_3G047140 & 1053 & 2 & 350 & WRKYGQK & $\mathrm{C} 2 \mathrm{H} 2$ & 1 & II d \\
\hline CsWRKY31 & CsaV3_4G001260 & 873 & 2 & 290 & WRKYGQK & $\mathrm{C} 2 \mathrm{H} 2$ & 1 & II e \\
\hline CsWRKY32 & CsaV3_4G003030 & 648 & 1 & 215 & WRKYGQK & $\mathrm{C} 2 \mathrm{H} 2$ & 1 & II c \\
\hline CsWRKY33 & CsaV3_4G006110 & 810 & 1 & 269 & WRKYGQK & $\mathrm{C} 2 \mathrm{H} 2$ & 1 & II e \\
\hline CsWRKY34 & CsaV3_4G006120 & 603 & 1 & 200 & WRKYGQK & $\mathrm{C} 2 \mathrm{H} 2$ & 1 & II c \\
\hline CsWRKY35 & CsaV3_4G006480 & 1068 & 2 & 355 & WRKYGQK & $\mathrm{C} 2 \mathrm{HC}$ & 1 & III \\
\hline CsWRKY36 & CsaV3_4G025110 & 840 & 2 & 279 & WRKYGQK & $\mathrm{C} 2 \mathrm{H} 2$ & 1 & II c \\
\hline CsWRKY37 & CsaV3_4G034570 & 1176 & 2 & 391 & WRKYGQK & $\mathrm{C} 2 \mathrm{H} 2$ & 1 & II d \\
\hline CsWRKY38 & CsaV3_4G036610 & 981 & 4 & 326 & WRKYGQK & $\mathrm{C} 2 \mathrm{H} 2$ & 1 & II a \\
\hline CsWRKY39 & CsaV3_5G001010 & 1035 & 2 & 344 & WRKYGQK & $\mathrm{C} 2 \mathrm{H} 2$ & 1 & II e \\
\hline CsWRKY40 & CsaV3_5G011060 & 639 & 1 & 212 & WRKYGQK & - & 1 & III \\
\hline CsWRKY41 & CsaV3_5G011080 & 849 & 2 & 282 & WRKYGQK & $\mathrm{C} 2 \mathrm{HC}$ & 1 & III \\
\hline CsWRKY42 & CsaV3_5G033090 & 1173 & 3 & 390 & WRKYGQK & $\mathrm{C} 2 \mathrm{H} 2$ & 1 & 1 \\
\hline
\end{tabular}


Table 1 Features of WRKY genes identified in cucumber (Continued)

\begin{tabular}{|c|c|c|c|c|c|c|c|c|}
\hline \multirow[t]{2}{*}{ Name } & \multirow[t]{2}{*}{ Gene ID (V3) } & \multirow[t]{2}{*}{ ORF } & \multirow{2}{*}{$\begin{array}{l}\text { Intron } \\
\text { number }\end{array}$} & \multirow[t]{2}{*}{ AA } & \multicolumn{3}{|l|}{ WRKY domain } & \multirow[t]{2}{*}{ Group } \\
\hline & & & & & Conserved heptapeptide & Zinc-finger type & $\begin{array}{l}\text { Domain } \\
\text { number }\end{array}$ & \\
\hline CsWRKY43 & CsaV3_5G038330 & 1521 & 3 & 506 & WRKYGQK/WRKYGQK & $\mathrm{C} 2 \mathrm{H} 2$ & 2 & 1 \\
\hline CsWRKY44 & CsaV3_6G013820 & 921 & 2 & 306 & WRKYGQK & $\mathrm{C} 2 \mathrm{H} 2$ & 1 & II c \\
\hline CsWRKY45 & CsaV3_6G018960 & 1287 & 4 & 428 & WRKYGQK/WRKYGQK & $\mathrm{C} 2 \mathrm{H} 2$ & 2 & 1 \\
\hline CsWRKY46 & CsaV3_6G028510 & 1359 & 4 & 452 & WRKYGQK & $\mathrm{C} 2 \mathrm{H} 2$ & 1 & II c \\
\hline CsWRKY47 & CsaV3_6G032100 & 360 & 3 & 119 & WRKYGKK & $\mathrm{C} 2$ & 1 & II c \\
\hline CsWRKY48 & CsaV3_6G032480 & 921 & 2 & 306 & WRKYGQK & $\mathrm{C} 2 \mathrm{H} 2$ & 1 & II c \\
\hline CsWRKY49 & CsaV3_6G042200 & 735 & 2 & 244 & WRKYGQK & $\mathrm{C} 2 \mathrm{H} 2$ & 1 & II c \\
\hline CsWRKY50 & CsaV3_6G042280 & 600 & 2 & 199 & WRKYGKK & $\mathrm{C} 2 \mathrm{H} 2$ & 1 & II c \\
\hline CsWRKY51 & CsaV3_6G043450 & 885 & 2 & 294 & WRKYGQK & $\mathrm{C} 2 \mathrm{H} 2$ & 1 & II d \\
\hline CsWRKY52 & CsaV3_6G048830 & 1872 & 5 & 623 & WRKYGQK & $\mathrm{C} 2 \mathrm{H} 2$ & 1 & II $\mathbf{b}$ \\
\hline CsWRKY53 & CsaV3_6G051490 & 786 & 2 & 261 & WRKYGQK & $\mathrm{C} 2 \mathrm{H} 2$ & 1 & II c \\
\hline CsWRKY54 & CsaV3_6G052610 & 1566 & 2 & 521 & WRKYGQK & $\mathrm{C} 2 \mathrm{H} 2$ & 1 & II e \\
\hline CsWRKY55 & CsaV3_7G002670 & 1302 & 4 & 433 & WRKYGQK/WRKYGQK & $\mathrm{C} 2 \mathrm{H} 2$ & 2 & 1 \\
\hline CsWRKY56 & CsaV3_7G003470 & 1608 & 4 & 535 & WRKYGQK & $\mathrm{C} 2 \mathrm{H} 2$ & 1 & II-b \\
\hline CsWRKY57 & CsaV3_7G005750 & 897 & 2 & 298 & WRKYGQK & $\mathrm{C} 2 \mathrm{H} 2$ & 1 & II e \\
\hline CsWRKY58 & CsaV3_7G022650 & 894 & 2 & 297 & WRKYGQK & $\mathrm{C} 2 \mathrm{H} 2$ & 1 & II c \\
\hline CsWRKY59 & CsaV3_7G025370 & 891 & 2 & 296 & WRKYGQK & $\mathrm{C} 2 \mathrm{HC}$ & 1 & III \\
\hline CsWRKY60 & CsaV3_7G027490 & 768 & 3 & 255 & WRKYGQK & $\mathrm{C} 2 \mathrm{H} 2$ & 1 & II d \\
\hline CsWRKY61 & CsaV3_7G030110 & 729 & 2 & 242 & WRKYGQK & $\mathrm{C} 2 \mathrm{H} 2$ & 1 & II $c$ \\
\hline
\end{tabular}

including eight dicots (Arabidopsis, castor bean, cucumber, grape, tomato, pear, potato, and poplar) and six monocots (barley, rice, maize, bread wheat, Brachypodium and millet). The WRKY domain sequence of these WRKY IIa genes was used to construct a phylogenetic tree via MEGA 5.0 (Additional file 5: Table S3).

As shown in the phylogenetic tree we constructed, the WRKY IIa proteins were categorized into seven clades (Fig. 3). WRKYs from the phylogenetically closer species clustered together in the same clade. For example, the members of clades 1 and 2 were all from dicots, whereas clades 4-7 contained proteins only from monocots; clade 3 was further divided into two different subclades (clades $3 \mathrm{a}$ and $3 \mathrm{~b}$ ) based on members from dicots or monocots, implying that the different evolutionary patterns of Group IIa WRKYs in dicots and monocots may have occurred after their divergence. WRKY members from one species clustered together at most within the three clades, and all the WRKY proteins that divided into three clades were from monocots. For the WRKYs from dicots, each of the four species (cucumber, grape, pear, and poplar) contributed at least one gene to clade 1 and clade 2; however, the three species (castor bean, tomato, and potato) clustered specifically within clade 1 or clade 2. These results suggested that numerous evolutionary splits and diversifications of WRKYS have occurred among different species.

\section{Gene structure and motif composition of CsWRKYs}

Gene structural diversity can reflect the evolution of multigene families [68]. Therefore, we analysed the exon-intron organization within the ORF (open reading fame) sequences of each CsWRKY gene (CsWRKY40, which lacked a zinc finger motif, was removed) to acquire more insight into the evolution of the WRKY family in cucumber. Previous studies showed that the majority of Populus and soybean WRKY members harboured two to four introns $[48,62]$. Consistently, more than $80 \%$ of the members of the CsWRKY genes contain two to four introns (seven with one intron, 29 with two introns, 10 with three introns, 12 with four introns, two with five introns, and one with six introns) (Fig. 4 and Table 1). As shown in Fig. 4, a greater number of introns were observed in Group I, which varied from three to six. All WRKY domains typically contain an intron, and the position of this intron is extremely highly conserved [57]. We found that all CsWRKYs contained an intron in their WRKY domains. This intron within the Groups I (the C-terminal WRKY domain), IIc, IId, IIe, and III WRKY genes had the same 


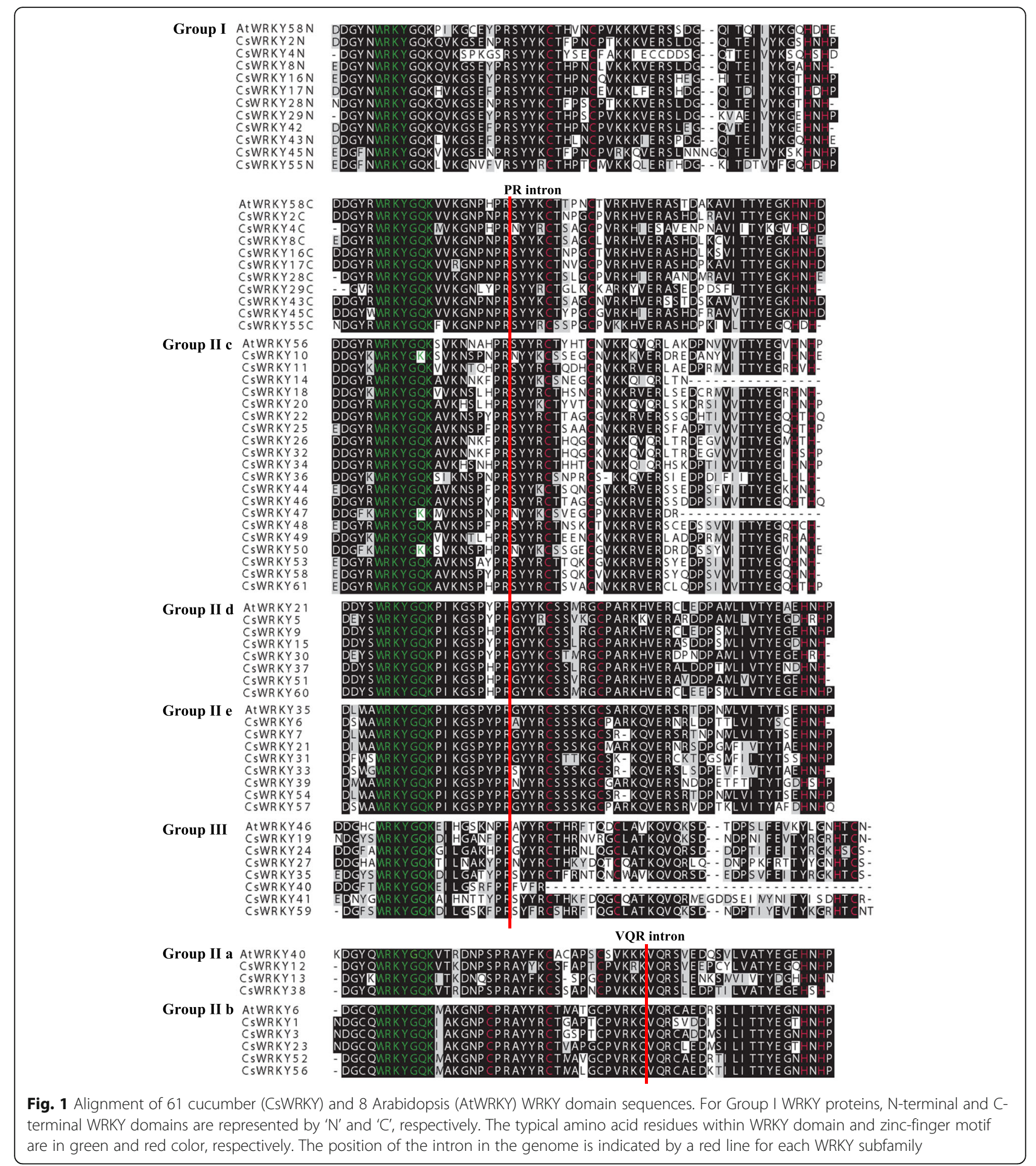

location, which was after the codons for the invariant PR amino acid sequence (PR intron) (Fig. 1). The VQR intron, which occurs before the invariant VQR amino acid sequence, was observed in the Group IIa and IIb genes.

To better understand the conservation and diversification of CsWRKYs, the putative motifs of all CsWRKY proteins were predicted by MEME motif analysis. As expected, the CsWRKYs that were categorized into the same group shared highly similar motif compositions (Fig. 4 and Additional file 6: Table S4). For instance, motif 9 was found to be specific to Groups IId and IIe, whereas motif 10 was unique to Groups IIb and IIc; Groups IIe and IIc contained only two or three motifs, 


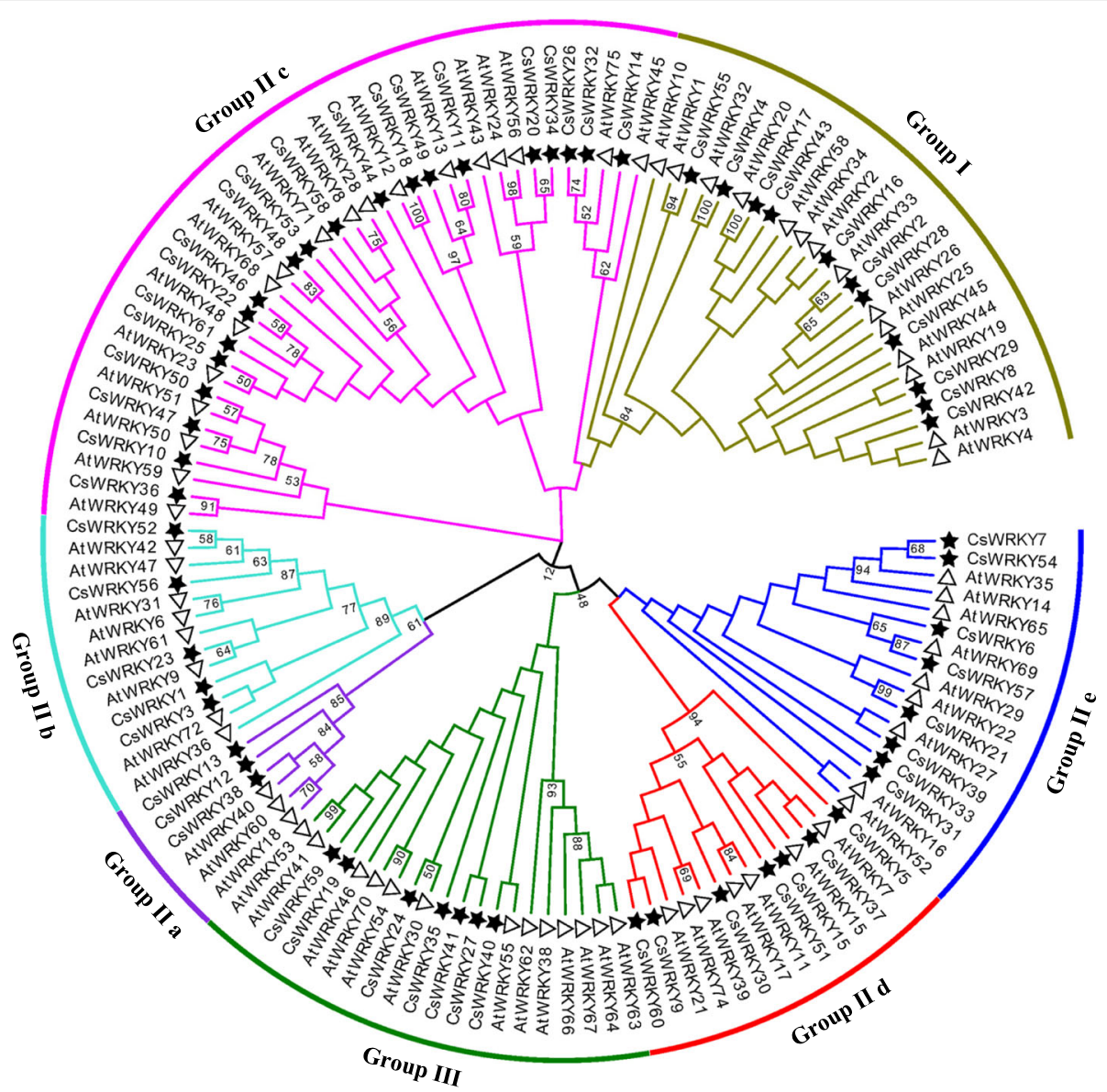

Fig. 2 The phylogenetic tree of the total WRKY proteins from cucumber and Arabidopsis. The WRKY domains were used for phylogenetic analysis by MEGA5 software with bootstrap test of 1000 times. The arcs with different color represent 7 subgroups of WRKY proteins. The black solid star and hollow triangle represent WRKY domain from cucumber and Arabidopsis, respectively

while Group IIb harboured 5 motifs. The functions of most of these motifs remain to be elucidated.

Overall, the closely related CsWRKYs in the phylogenetic tree shared similar gene structural and common motif compositions, suggesting that the CsWRKYs within the same group may play similar functional roles.

\section{Synteny analysis of CsWRKY genes}

The segmental duplication events occurring in the cucumber WRKY family were investigated by conducting a synteny analysis of the CsWRKY genes using BLASTP and MCScanX. As shown in Fig. 5, 14 segmental duplication events involving 25 WRKY genes were observed (Additional file 7: Table S5). In contrast, tandem duplication events, which were defined by a chromosomal region within $200 \mathrm{~kb}$ containing two or more genes, were not identified for cucumber WRKY genes. These results suggested that some CsWRKYs were possibly generated by segmental duplication events and that the evolution of CsWRKY genes may have been driven, at least in part, by segmental duplication events.
The phylogenetic mechanisms of the cucumber WRKY family were further explored by constructing comparative syntenic maps of cucumber associated with five representative species, including three dicots (Arabidopsis, tomato and watermelon) and two monocots (rice and maize) (Fig. 6). Fifty-two, 29, 27, 9, and 5 CsWRKY genes showed syntenic relationships with those in the other five species: watermelon, tomato, Arabidopsis, rice and maize, respectively. A total of 52 WRKY collinear gene pairs between cucumber and watermelon were identified, followed by cucumber and Arabidopsis (41), cucumber and tomato (37), cucumber and rice (9), and cucumber and maize (7) (Additional file 8: Table S6). Both cucumber and watermelon belong to the gourd family, and more than $85 \%$ of the CsWRKY genes showed a syntenic relationship with WRKYS in watermelon, and one CsWRKY gene was associated with only one syntenic gene pair, indicating that WRKY genes in cucumber and watermelon evolved from the same ancient WRKY genes. CsWRKY21 and CsWRKY28 were found to be associated with two syntenic gene pairs 
Table 2 Summary of the number of WRKY proteins in diverse plant species

\begin{tabular}{|c|c|c|c|c|c|c|c|c|c|c|c|}
\hline \multirow[t]{2}{*}{ Species } & \multirow[t]{2}{*}{ Name } & \multirow[t]{2}{*}{ Total } & \multicolumn{7}{|c|}{ Group } & \multirow[t]{2}{*}{ NG } & \multirow[t]{2}{*}{ References } \\
\hline & & & I & Ila & $\| \mathrm{b}$ & ॥c & Ild & lle & III & & \\
\hline Ananas comosus & AcWRKY & 54 & 12 & 2 & 7 & 13 & 7 & 5 & 8 & 0 & {$[22]$} \\
\hline Arabidopsis thaliana & AtWRKY & 72 & 13 & 4 & 7 & 18 & 7 & 9 & 14 & 0 & {$[16]$} \\
\hline Brachypodium distachyon & BdWRKY & 86 & 15 & 3 & 6 & 21 & 6 & 10 & 23 & 2 & {$[57]$} \\
\hline Brassica napus & BnWRKY & 300 & 78 & 11 & 34 & 55 & 28 & 30 & 51 & 13 & [47] \\
\hline Cucumis sative & CsWRKY & 61 & 14 & 3 & 5 & 17 & 7 & 8 & 7 & 0 & This study \\
\hline Glycine max & GmWRKY & 188 & 32 & 14 & 33 & 42 & 21 & 20 & 26 & 0 & {$[48]$} \\
\hline Gossypium raimondii & GrWRKY & 112 & 20 & 7 & 16 & 26 & 16 & 13 & 14 & 0 & {$[58]$} \\
\hline Gossypium arboreum & GaWRKY & 109 & 19 & 7 & 16 & 26 & 14 & 13 & 14 & 0 & {$[58]$} \\
\hline Hordeum vulgare & HvWRKY & 45 & 8 & 4 & 1 & 11 & 5 & 3 & 13 & 0 & [59] \\
\hline Manihot esculenta & MeWRKY & 85 & 17 & 5 & 14 & 20 & 8 & 9 & 12 & 0 & {$[60]$} \\
\hline Musa acuminate & MusaWRKY & 153 & 14 & 7 & 11 & 15 & 15 & 13 & 6 & 72 & [61] \\
\hline Oryza sativa & OsWRKY & $103 a$ & 15 & 4 & 8 & 15 & 7 & 11 & 36 & 0 & [17] \\
\hline Populus trichocarpa & PtrWRKY & 100 & 22 & 5 & 9 & 27 & 13 & 13 & 10 & 1 & {$[62]$} \\
\hline Pyrus bretschneideri & PbrWRKY & 103 & 17 & 6 & 10 & 24 & 15 & 16 & 15 & 0 & {$[63]$} \\
\hline Ricinus communis & RcWRKY & 47 & 9 & 3 & 10 & 12 & 3 & 5 & 5 & 0 & [64] \\
\hline Sesamum indicum & SiWRKY & 71 & 12 & 4 & 11 & 18 & 7 & 8 & 7 & 4 & {$[65]$} \\
\hline Solanum lycopersicum & SIWRKY & 81 & 15 & 5 & 8 & 16 & 6 & 17 & 11 & 3 & [19] \\
\hline Solanum tuberosum & StWRKY & 82 & 14 & 6 & 5 & 16 & 15 & 12 & 14 & 0 & {$[66]$} \\
\hline Triticum aestivum & TaWRKY & 160 & 20 & 16 & 3 & 34 & 17 & 11 & 57 & 2 & [51] \\
\hline Vitis vinifera & VVWRKY & 72 & 15 & 5 & 7 & 22 & 8 & 8 & 7 & 0 & {$[67]$} \\
\hline Zea mays & ZmWRKY & $120 \mathrm{~b}$ & 15 & 6 & 8 & 22 & 13 & 16 & 30 & 0 & [18] \\
\hline
\end{tabular}

a98 WRKY genes in japonica and 102 in indica rice. bother 10 ZmWRKY genes were classied in Group II. NG no group

between cucumber and tomato/rice/maize; some CsWRKY genes were associated with three collinear gene pairs (between cucumber and tomato/Arabidopsis WRKY genes), speculating that these CsWRKYs may play an important role in the evolution of the WRKY gene family. Importantly, collinear CsWRKY21 gene pairs were observed between cucumber and all of the other five species, suggesting that this orthologous pair may have formed before the divergence of dicotyledonous and monocotyledonous plants.

\section{CsWRKYs expression profiles in different organs}

The expression patterns of all 61 CsWRKYs were investigated using a standard transcriptome analysis procedure based on public transcriptomic data of different tissues of cucumber, including roots, stems, leaves, female flowers, male flowers, ovaries, expanded unfertilized ovaries, expanded fertilized ovaries, and tendrils [69]. Among the 61 CsWRKY genes, 41 CsWRKYs were expressed in all detected samples (FPKM $>0$ ), and 24 genes showed constitutive expression (FPKM $>1$ in all samples) (Additional file 9: Table S7). Some CsWRKY genes showed preferential expression across all tissues tested. Nineteen genes in the roots, two genes in the tendrils (CsWRKY50/59), and two genes in the female flowers (CsWRKY48/12) exhibited the highest transcript levels. The expression analysis of the different fruit developmental stages showed that several genes (CsWRKY9/40/54) had higher expression in the ovaries than in the expanded ovaries (fertilized and unfertilized). In addition, the transcript levels of some CsWRKYs (such as CsWRKY19/27/41/57) decreased in the fertilized expanded ovaries (Fig. 7). These results indicated that these genes may play roles in many aspects of cucumber plant development, including ovary development and fruit fertilization.

\section{Expression patterns of CsWRKYs in response to abiotic and biotic stresses}

To confirm whether CsWRKYs are involved in the response to various stresses, we analysed the comprehensive expression patterns of CsWRKY genes under different abiotic and biotic stresses, including salt, heat, downy mildew (DM, Pseudoperonospora cubensis) and powdery mildew (PM, Podosphaera fusca), based on public transcriptome information [54, 70, 71] and transcriptomic data that we generated. 


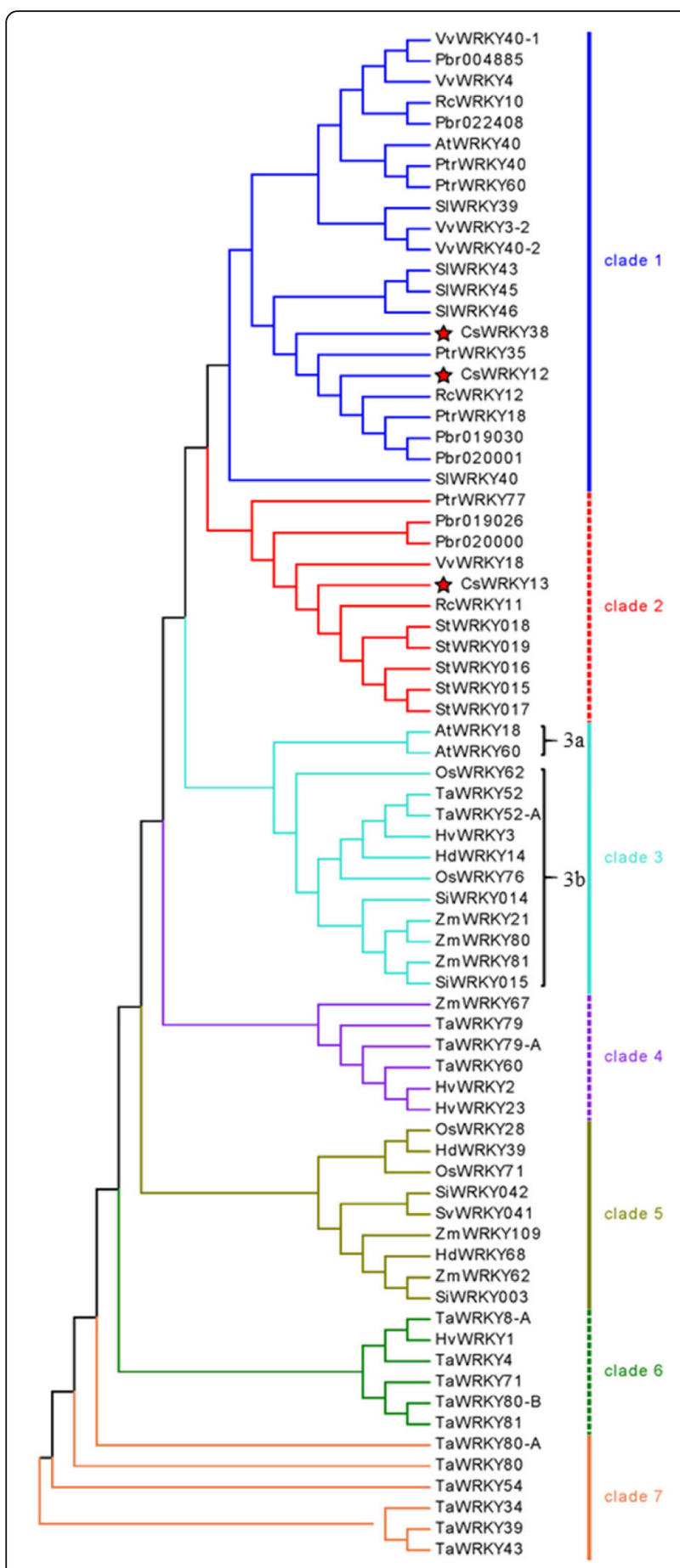

Fig. 3 Phylogenetic clustering of group lla WRKY proteins among fourteen different plant species. The phylogenetic tree was constructed by MEGA 5.0 using the Neighbor-Joining method. The WRKYs are classified into seven main clades with two subclades. The differentcolored branch represents different clades. The red solid star indicates group lla WRKY proteins from cucumber

To investigate the potential functions of CsWRKYs in resistance to salt stress, we performed a CsWRKYs expression analysis after salt treatment based on public transcriptomic data [70] (Additional file 10: Table S8). We observed that the expression levels of CsWRKY27, CsWRKY41 and CsWRKY50 considerably increased in response to salt stress. Moreover, seven genes exhibited the opposite trend with exposure to salt stress (Fig. 8a). Previous studies have shown that Silicon (Si) application can improve plant growth under salt stress [72]. Among the seven genes that were downregulated under salt stress, the expression levels of six genes reverted to normal expression levels, and the DEGs (differentially expressed genes) were all upregulated in response to exogenous $\mathrm{Si}$ treatment, implying a potential role of these WRKY genes in the Si-based alleviation of salt stress (Fig. 8a). Furthermore, the expression patterns of all 61 CsWRKY genes in the transcriptomic data, which were derived from leaves subjected to different heat treatment durations, were investigated in this study (Additional file 10: Table S8). Correlation and cluster analyses were used to explore the similarity among the transcriptomes. Two samples (HT3h_2 and HT6h_2) were removed due to their poor uniformity, and the remaining seven samples were used for the following analysis (Additional file 11: Fig. S3). As shown in Fig. 8b, 21 CsWRKY genes were significantly induced/repressed by heat stress. The variation trend of the expression of most WRKY genes in response to heat stress for $3 \mathrm{~h}$ (hours) was consistent with that for $6 \mathrm{~h}$. Overall, the transcript levels of five CsWRKY genes (CsWRKY27/41/50/52/57) were significantly affected by both salt and heat stress treatments.

To explore the potential functions of CsWRKYs in resistance to biotic stresses, we performed a CsWRKYs expression analysis of the susceptible and resistant cucumber lines inoculated with PM for $48 \mathrm{~h}$ based on published RNA-seq data [71] (Fig. 9a and Additional file 10: Table S8). Eleven and 12 CsWRKY genes that were differentially expressed were identified in the susceptible and resistant cucumber lines, respectively, compared with the controls. These results indicated that these WRKYs may be influenced by PM stress. The expression patterns of CsWRKY10 and CsWRKY50 were opposite in the susceptible and resistant cucumber lines under inoculation with PM, implicating the important role of these two WRKY genes in the response to PM infection. The expression of CsWRKY genes in response to DM infection was obtained by transcriptome analysis based on RNA-seq data published by Adhikari et al. [54] (Additional file 10: Table S8). Twenty-five CsWRKY genes in cucumber were involved in responses to DM infection, indicating that they were induced to play a role in response to DM infection (Fig. 9b). We identified 12 CsWRKY genes (CsWRKY10/14/19/27/28/32/35/46/ $50 / 52 / 59 / 61)$ that were differentially expressed in response to the inoculation of PM and DM (Fig. 9), 


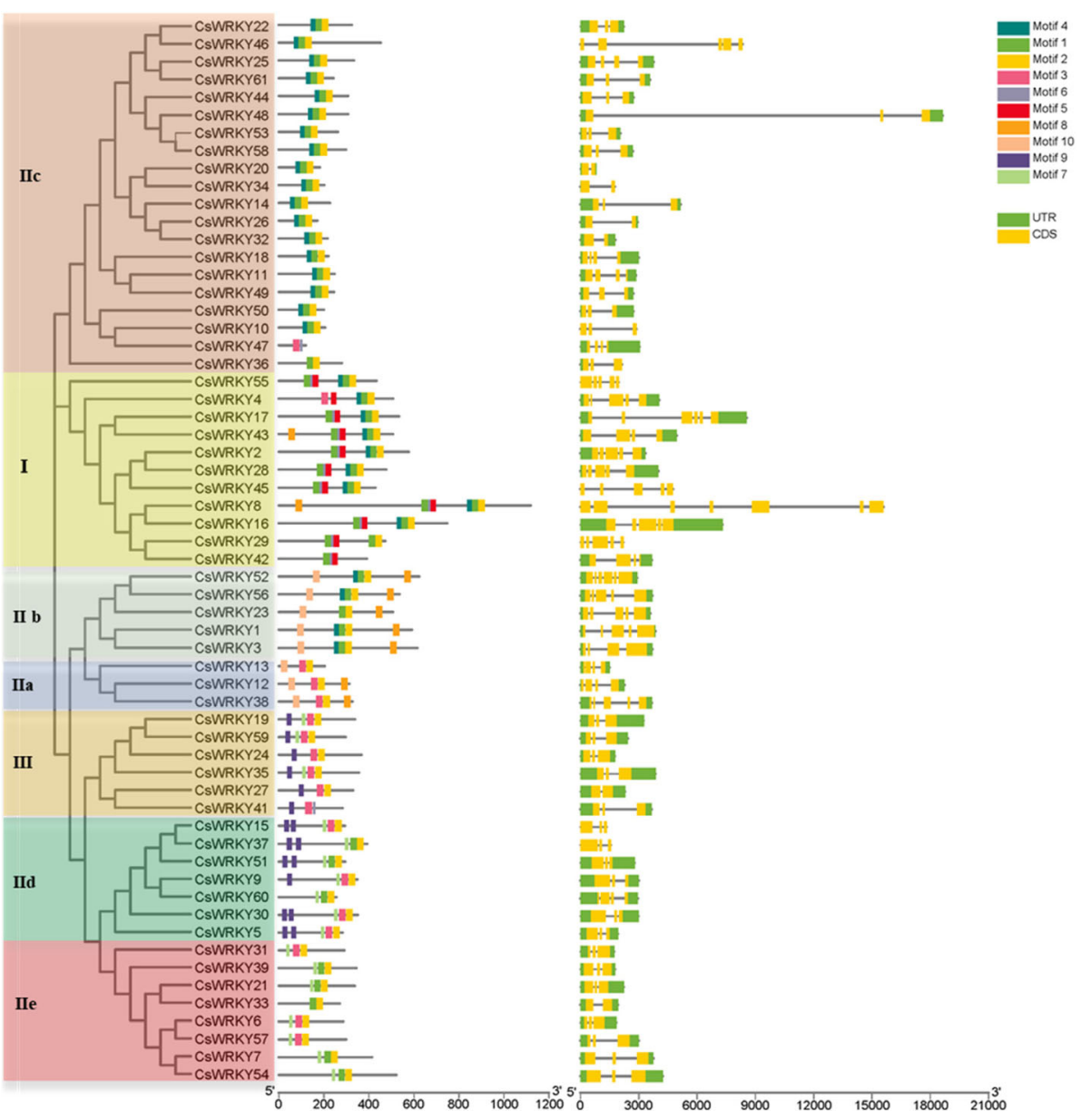

Fig. 4 Phylogenetic clustering, conserved protein motifs and gene structure of CsWRKY genes. Left panel: the phylogenetic tree was constructed from the WRKY domain sequences of CsWRKY proteins. The different groups and subgroups are shown in different colors. Middle panel: the motifs are represented by different colored boxes. Details of each motif are shown in Additional file 5. Right panel: gene structure of CsWRKY transcription factors. Untranslated 5'- and 3'-regions, exons, and introns are indicated by green boxes, yellow boxes, and black lines, respectively

indicating that these genes may play key roles in responses to biotic stresses. Some CsWRKY genes were affected only by inoculation of PM and/or DM and not by abiotic (heat and salt) stresses (Figs. 8 and 9). For instance, CsWRKY46 was expressed significantly in response to inoculation of PM and DM but not to salt and heat stresses; moreover, CsWRKY15 was not induced/repressed by any of the tested treatments except inoculation of PM. In addition, the expression levels of several CsWRKY genes were significantly affected by both abiotic stresses and biotic stresses (Figs. 8 and 9). For instance, CsWRKY27, CsWRKY50 and CsWRKY52 simultaneously responded to all treatments analysed, and the expression of CsWRKY59 was affected by all tested treatments except salt treatment.

\section{Discussion}

Although WRKY genes had been identified in the cucumber (9930) genome (v1.0) [55], it is essential to re- identify them. Because the information of the WRKY genes identified in v1.0 were no longer available, due to the elimination of v1.0 from CuGenDB (http://cucurbitgenomics.org/), whereas the assemblies (v2.0 and v3.0) were available. Therefore, we identified and characterized the WRKY family in the cucumber (9930) genome (v3.0). It is composed of 61 members, which were designated CsWRKY1 to CsWRKY61 on the basis of their chromosomal location; this number is higher than that identified in a previous study (57 WRKY genes) [55]. Compared with these previously reported CsWRKY genes, nine new CsWRKY genes were mapped onto the chromosomes, and five previous CsWRKY genes that could not be conclusively mapped to any chromosome were considered obsolete according to the current version of the cucumber genome (v3.0) (Additional file 1: Fig. S1 and Additional file 2: Fig. S2).

Based on the gene structures, amino acid sequences, conserved structural domains and phylogenetic 


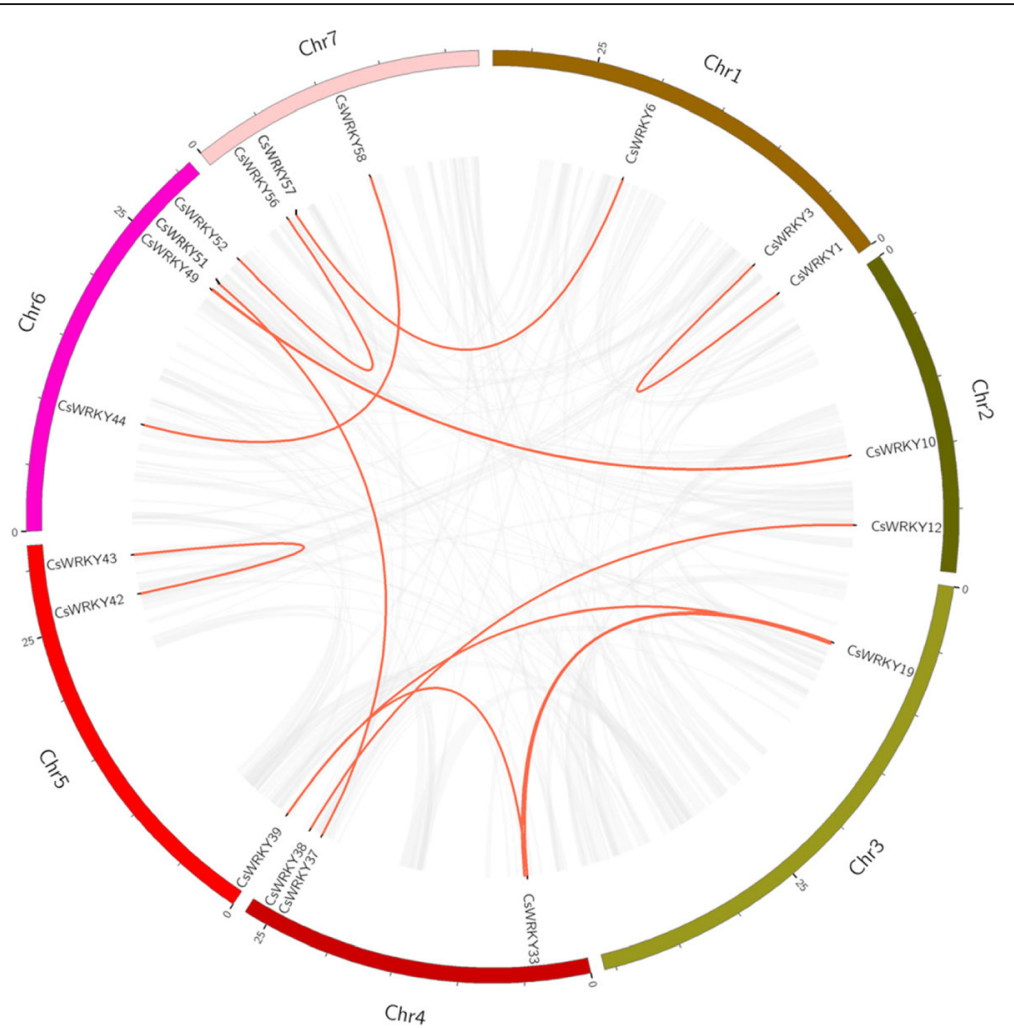

Fig. 5 Schematic representations for the interchromosomal relationships of CsWRKYs. Gray lines show synteny blocks in the cucumber genome, duplicated WRKY gene pairs are connected with red lines

relationships with $A$. thaliana, the 61 CsWRKY proteins were similar to the typical WRKY family proteins in other species with classifications into Groups I, IIa, IIb, IIc, IId, IIe and III (Table 1, Figs. 1 and 2). Rinerson et al. [20] proposed that there were four major WRKY TF lineages in flowering plants, Groups I + IIc, Groups IIa + IIb, Groups IId + IIe, and Group III, accurately reflecting the evolution of the WRKY family. This was also verified in cucumber; for example, the members of Groups IIa and IIb (or Groups IId and IIe) were divided into two subclades, which involved the same clade; some WRKY TFs from Group IIc were classified into one subclade together with the members of Group I (Fig. 2). Three CsWRKY proteins (CsWRKY10/47/50) in Group IIc showed sequence variation in their WRKY domains. Domain loss, which seems to be common in monocotyledons, is one of the divergent forces for expansion of the WRKY gene family [73, 74]; however, these loss-ofdomain events occur less for dicotyledons than for monocotyledons. For example, Group I contains one protein (AtWRKY10) having only one WRKY domain in Arabidopsis [74]. In cucumber, except CsWRKY42, all WRKY proteins of Group I have two WRKY domains, and the event by which the zinc finger motif was lost was also found in three WRKY TFs (CsWRKY14/40/47) (Fig. 1 and Table 1). According to previous studies, both the heptapeptide motif WRKYGQK and the zinc finger motif are required for the high binding affinity of WRKY TFs to their cognate cis-acting W-box element (TTGA $\mathrm{CC} / \mathrm{T})$. Therefore, variations in the heptapeptide motif and loss of the zinc finger motif might influence normal interactions of CsWRKYs with target genes, and it might be worth further studying the binding specificities and functions of these five CsWRKY proteins.

Both tandem duplication and chromosomal/segmental duplications contributed to the expansion of the WRKY gene family [24]. Comparison of the number of WRKY genes in the cucumber genome with other sequenced dicotyledon genomes showed that cucumber has fewer genes (Table 2). Fourteen segmental duplication events within 25 WRKY genes were observed (Fig. 5 and Additional file 7: Table S5), while tandem duplication events were lacking. Therefore, the lack of tandem duplication might be a possible reason for the smaller number of $C s W R K Y$ genes, and segmental duplication was a major driver of WRKY gene expansion during the cucumber evolutionary process. Moreover, we identified that more than $85 \%$ (52 of 61) of CsWRKY genes showed orthologous relationships with $C l W R K Y$ genes (Additional file 8: Table S6), indicating that the segmental duplication of WRKY genes might have occurred in diploid progenitors before the divergence of the cucumber and watermelon. 


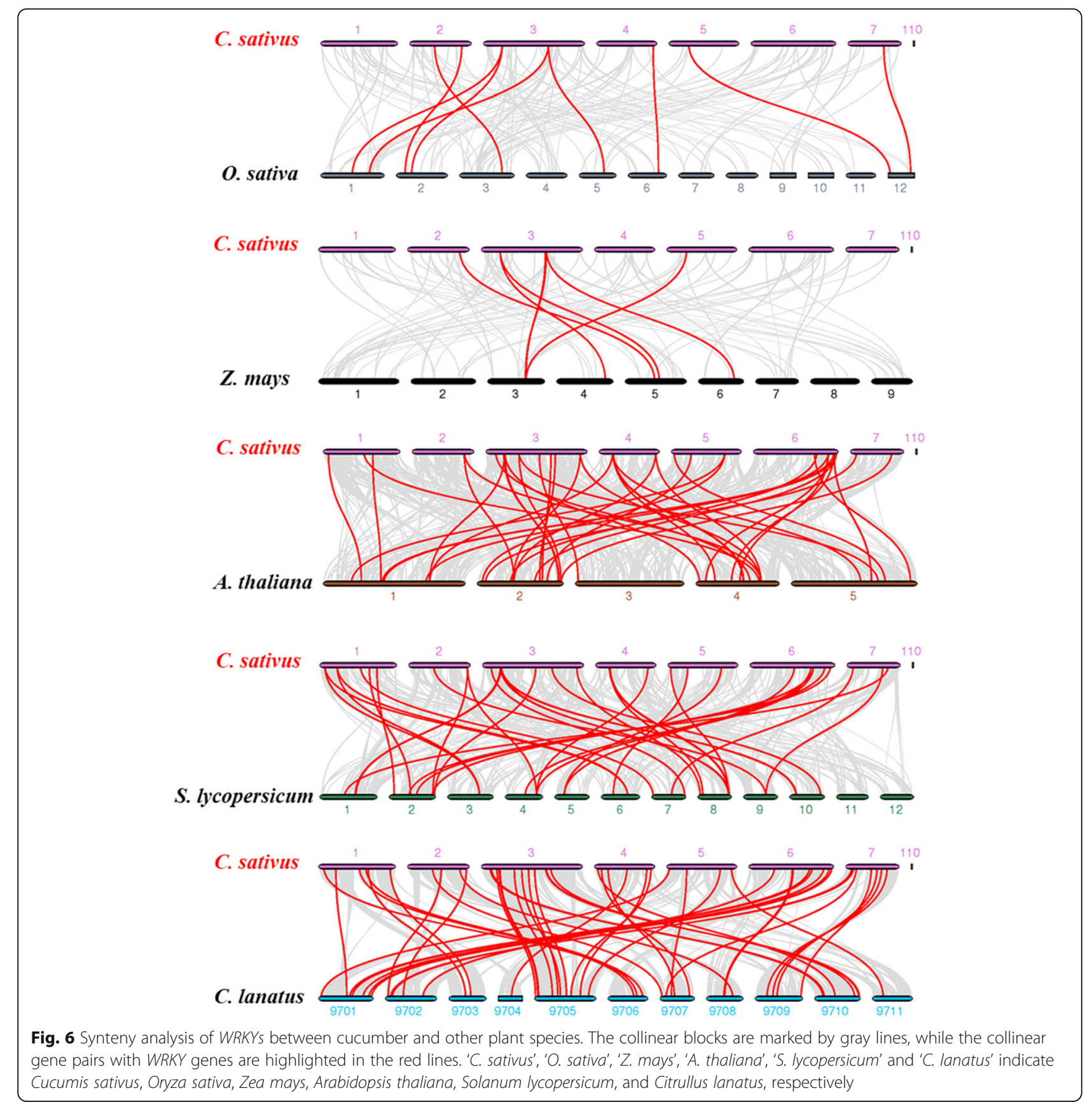

In 2015, Rinerson et al. used the genome sequences of a moss to propose the hypothesis that Group III genes were not the last to evolve; rather, Group IIa genes were [20]. Among the WRKY gene family, Group IIa genes compose the subclade with the smallest number of members and appear to play many important roles in the response to different stresses [20]. The availability of increased numbers of Group IIa members of sequenced plant genomes could provide additional clues about the evolution of the WRKY TF family. In this study, we found that members of the plant WRKY Group IIa from closely related species tended to be clustered together, and there were monocot (clades 1, 2 and 3a)- and dicot (clades 3b and 4-7)-specific clades (Fig. 3). These results suggested that WRKY IIa genes might have evolved independently after the divergence of monocots and dicots.

It is well known that gene expression is correlated with gene function [75]. In this study, the expression pattern of all 61 CsWRKY genes was analysed in nine different tissues of cucumber, including the roots, stems, leaves, flowers, ovaries, and tendrils. We found that 19 CsWRKY genes were expressed specifically in the roots 


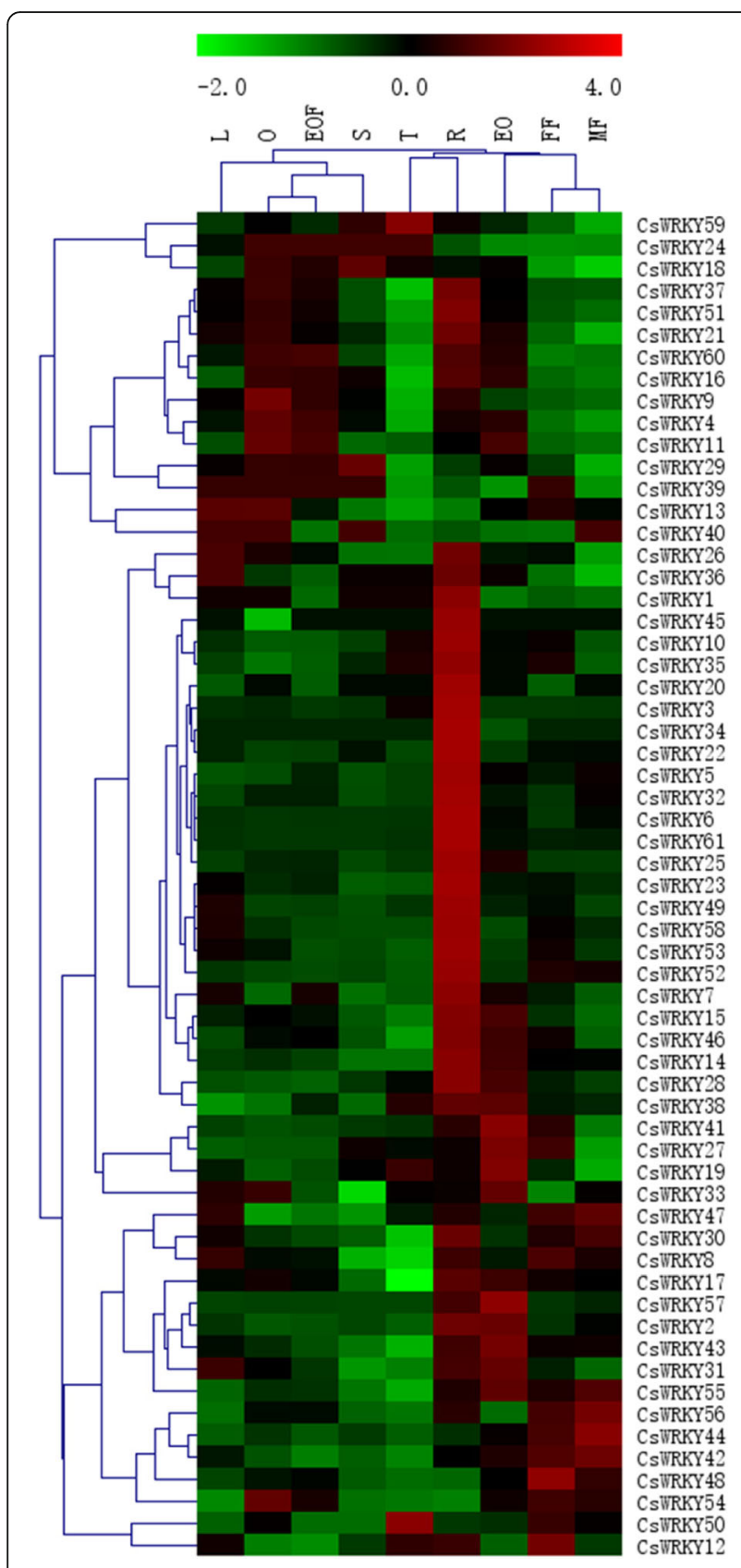

Fig. 7 Tissue-specific expression of WRKY genes in cucumber. The transcriptional levels of CSWRKY genes in nine tissues of cucumber 9930 were investigated based on a public transcriptome data, and only one biological replication was used for each tissue sample [69]. The genome-wide expression of CSWRKY genes was shown on a heatmap using FPKM value, and the expression data were genewisely normalized by MeV (Multiple Experiment Viewer) software. -2.0 to 4.0 was artificially set with the color scale limits according to the normalized value. The color scale shows increasing expression levels from green to red. L, leaves; O, ovary; EOF, expanded fertilized ovary; S, stem; T, tendril; R, root; EO, expanded unfertilized ovary; FF, female flower; MF, male flower
(Fig. 7). As previously reported, AtWRKY23, AtWRKY75 and AtWRKY6 were found to regulate root development $[76,77]$, and their close genetic homologous genes in cucumber, CsWRKY25, CsWRKY32 and CsWRKY52, respectively, were expressed specifically in the roots. According to these results, all genes expressed specifically in the roots were assumed to be key regulators of root development and may play roles in response to various stresses that first affect plants below ground. Additionally, CsWRKY50 and CsWRKY59 were highly expressed in the tendrils, which are considered abnormal leaves in cucumber, implying that they may regulate leaf morphogenesis in cucumber. Noticeably, the expression profiles of cucumber WRKY genes in this study were not consistent with the results in the study of Ling et al. [55]. For example, WRKY18 and WRKY56 have extremely different patterns of expression profiles between these two studies (Fig. 7) [55]. The reason might be that although they have the same name, they actually are different genes. This prediction could be supported by that WRKY56 could not be conclusively mapped to any chromosome in the previous study [55], but to chromosome 7 in our study (Additional file 1: Fig. S1), and that WRKY18 was localized in different position on chromosome 3 (Additional file 1: Fig. S1) [55]. However, now it is very difficult to clarify the reason because that the v1.0 of the cucumber genome sequences is not available yet, and Ling et al. did not provide the gene or protein sequences of WRKYs in their paper [55].

WRKY proteins constitute one of the most important TF families and are involved in responses to biotic and abiotic stresses [25]. At least 26 and 54 WRKY genes were identified to respond to abiotic stress in Arabidopsis and rice, respectively $[38,78]$. In this study, we further explored the expression of 61 CsWRKY genes under multiple stresses. Most of them were induced/repressed by at least one of the stresses that we tested (heat, salinity, and inoculation of DM and PM), indicating that the CsWRKYs play crucial roles in cucumber stress responses. Four CsWRKY genes, CsWRKY9, CsWRKY18, CsWRKY48 and CsWRKY57, were responsive to heat and/or salinity stress but not to the inoculation of DM and PM (Figs. 8 and 9). Previous studies have revealed that one WRKY gene can function in response to several stresses. For example, overexpressing AtWRKY30 improved tolerance to oxidative and salinity stresses during seed germination [79]. Among these four genes, the transcript level of CsWRKY57 was significantly affected by both salt and heat stress treatments (Fig. 8), suggesting that this gene acts as the most important gene to regulate susceptibility to abiotic stresses in cucumber. Correspondingly, 10 Cs WRKY genes (CsWRKY1/3/4/15/ $21 / 22 / 30 / 46 / 53 / 58$ ) were affected only by inoculation of PM and/or DM and not by abiotic (heat and salt) 


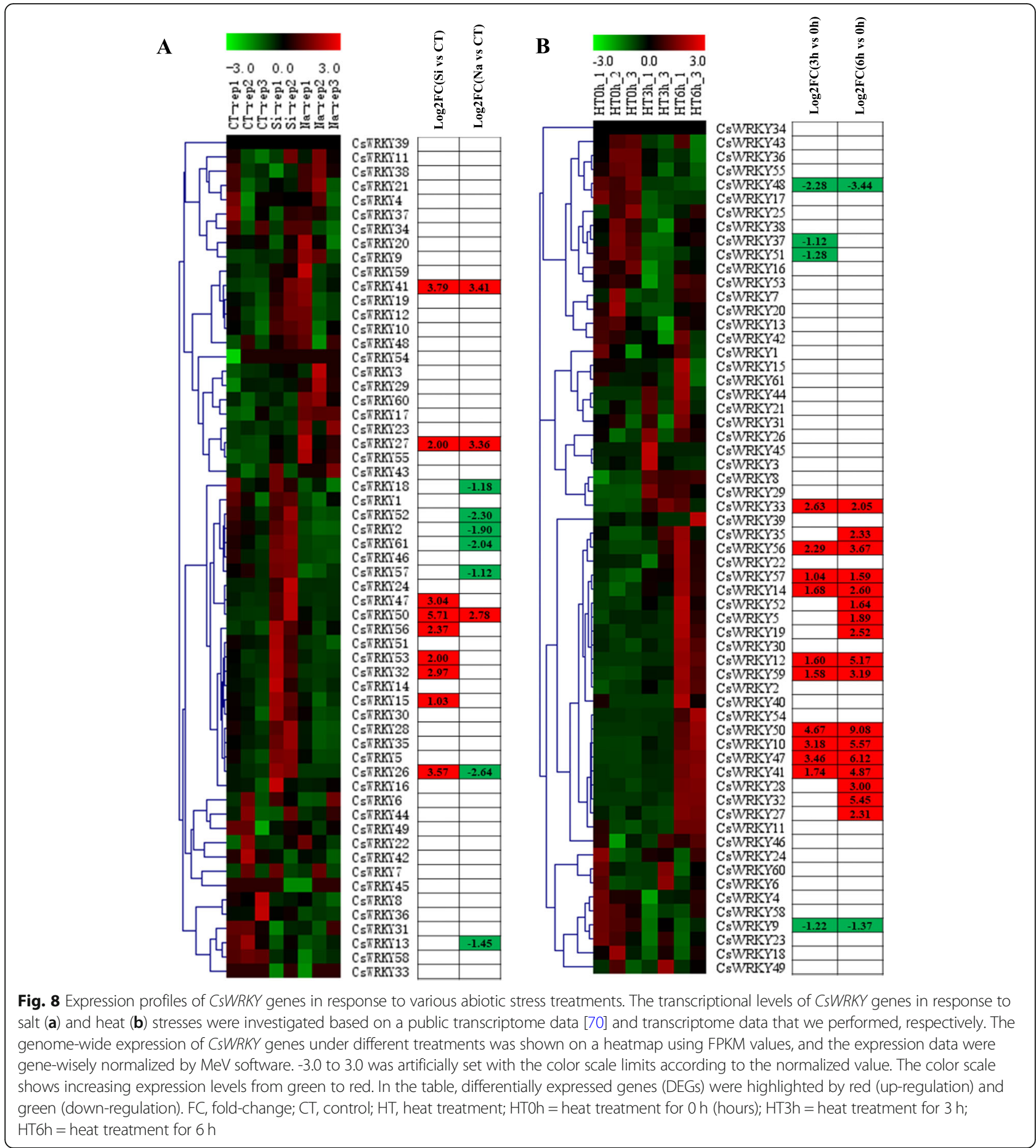

stresses (Figs. 8 and 9), and only Cs WRKY46 was observed to be differentially expressed in response to inoculation of PM and DM. These results revealed significant differences in the stress-induced expression of WRKYS in response to abiotic and biotic stresses. In addition, 22 CsWRKY genes were significantly affected by both abiotic stresses and biotic stresses (Figs. 8 and 9), indicating that some CsWRKY genes have similar functions in response to both abiotic and biotic stresses. For instance, the expression of CsWRKY59 was affected by all tested treatments except salt treatment; CsWRKY27, CsWRKY50 and CsWRKY52 simultaneously responded to all treatments that we analysed. The expression of OsWRKY67 was activated by rice blast inoculation; overexpression of OsWRKY67 in rice plants enhanced resistance to leaf blast, panicle blast and bacterial blight [80]; and its orthologue in cucumber, 


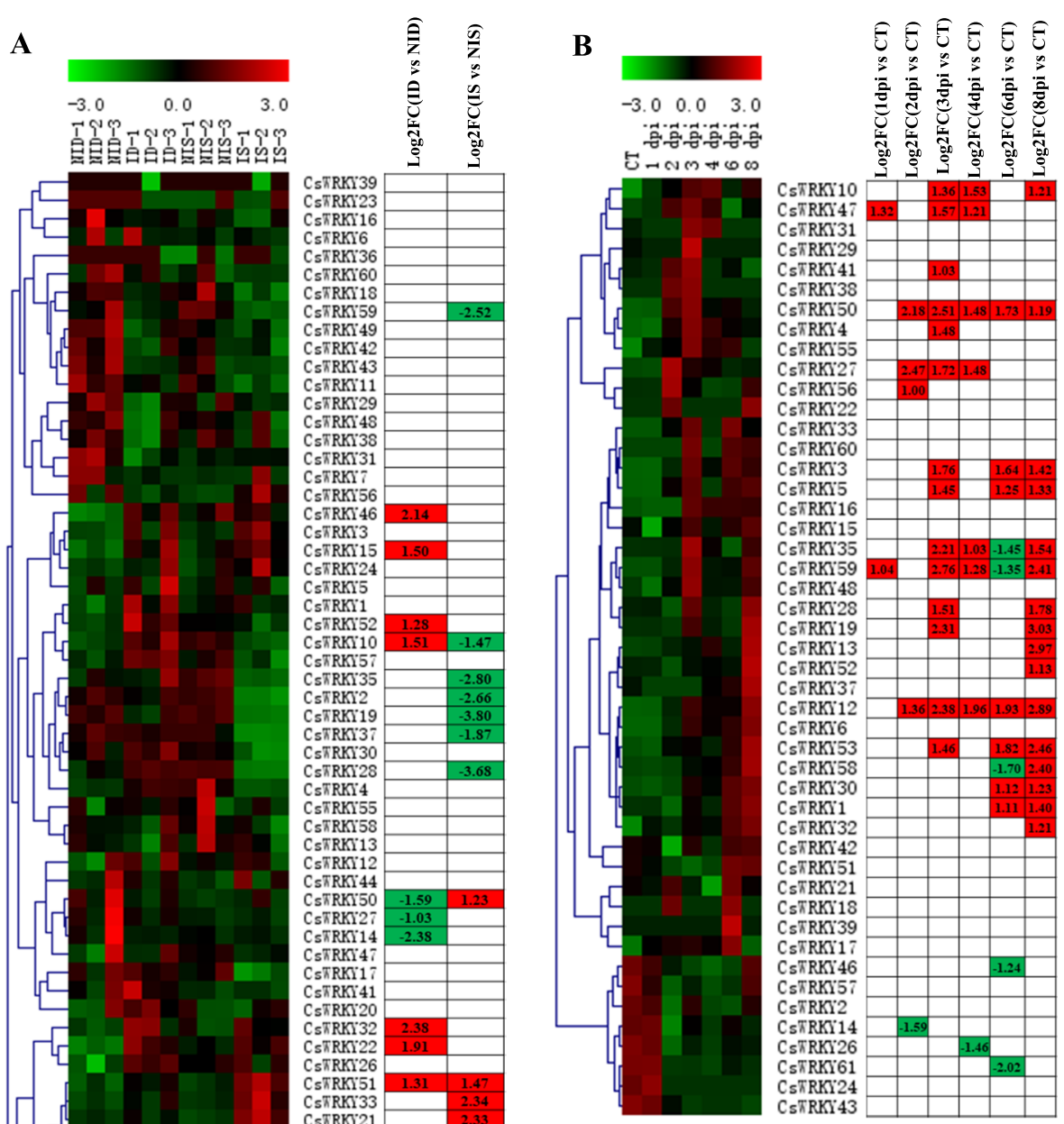

Fig. 9 Expression analysis of CSWRKYS under biotic stresses. The CSWRKY genes transcripts were determined after the infection of powdery mildew (PM) for $48 \mathrm{~h}$ (a) [71] and of downy mildew (DM) for 1 to 8 days post inoculation (b) [54], respectively. Without inoculation as the control (CT). The FPKM values of CSWRKY genes under different treatments were gene-wisely normalized and shown on a heatmap by MeV software. - 3.0 to 3.0 was artificially set with the color scale limits according to the normalized value. The color scale shows increasing expression levels from green to red. In the tables, red shadings indicated up-regulated genes, green shading indicated down-regulated genes. FC, fold-change; ID, DM inoculated susceptible cucumber line D8 leaves; NID, non-inoculated D8 leaves; IS, DM inoculated resistant cucumber line SSL508-28 leaves; NIS, non-inoculated SSL508-28 leaves; DPI, day post inoculation

CsWRKY50, was also induced by biotic stresses, suggesting the potential value of CsWRKY27, CsWRKY50 and CsWRKY52 in improvements to cucumber abiotic and biotic stress tolerance. Moreover, the expression of 12 CsWRKY genes (CsWRKY6/16/17/24/29/31/38/ $39 / 42 / 43 / 55 / 60$ ) was not observed in response to either the biotic stresses or abiotic stresses we analysed in this study.

As shown in Fig. 9, the expression of CsWRKY19 was downregulated by PM infection but upregulated by DM. The results indicated that WRKY genes might play different roles under different stress responses. Further analysis showed that responses to stresses occurred at different timepoints. CsWRKY10 and CsWRKY47 responded to heat stress at $3 \mathrm{~h}$, whereas the expression of CsWRKY28 and CsWRKY35 was affected at $6 \mathrm{~h}$ after heat stress began; CsWRKY56 was highly expressed only at $2 \mathrm{dpi}$, while infection by DM upregulated the expression of CsWRKY12 and CsWRKY50 between 2 to $8 \mathrm{dpi}$, suggesting that $C s W R K Y$ genes might play important regulatory roles at different stages in cucumber abiotic and biotic stress tolerance. 
Overall, these above findings provide insights into the potential functions of cucumber WRKY genes. The differential expression in response to different stresses indicated their functional diversification. Some CsWRKY genes might specifically respond to biotic or abiotic stress, while several genes may respond to both biotic and abiotic stress. In addition, some CsWRKY genes might not be involved in stress responses. These results are helpful for future functional characterization of CsWRKY genes and for the genetic improvement of the abiotic and biotic stress resistance of cucumber.

\section{Conclusions}

In the present study, 61 cucumber WRKY genes were identified, and a comprehensive analysis of those CsWRKY genes was carried out. First, the chromosomal location, conserved motifs, evolutional relationships and gene structure of the cucumber WRKY genes were examined. The expression patterns of the CsWRKY genes in nine different tissues of cucumber cultivar 9930 and in response to various stresses then showed that these genes may play important roles in cucumber growth and development. Furthermore, our results revealed differences and similarities in the stress-induced expression of CsWRKYs in response to abiotic and biotic stresses. In conclusion, our study provided a foundation for future studies into the functions of WRKY genes important in responses to abiotic and biotic stresses and the identification of new sources of resistance for breeding programmes.

\section{Methods}

\section{Gene identification and chromosomal locations}

The hidden Markov model (HMM) file of the WRKY domain (PF03106), downloaded from the Pfam protein family database (http://pfam.sanger.ac.uk/), was used for the identification of WRKY genes from the cucumber genomic database (v3.0) by HMMER 3.0. The default parameters were employed, and the cutoff value was 0.01. All CsWRKY genes that were queried from the cucumber genomic data based on the HMMER results were further examined to confirm the existence of the WRKY domain sequences through the Pfam (http://pfam.xfam.org/search\#tabview=tab1) and SMART (http://smart.embl-heidelberg. $\mathrm{de} /$ ) databases. We then manually examined each candidate gene to ensure the conserved heptapeptide sequence within the predicted WRKY domain and used PCR amplification and sequencing to further validate select CsWRKY genes. Sixty-one WRKY genes were ultimately identified and mapped to cucumber chromosomes according to their physical location information from the cucumber genomic database. The subcellular localization of CsWRKY proteins was predicted using CELLO (http://cello.life.nctu.edu.tw/).

\section{CsWRKY genes structure analysis, classification and phylogenetic analysis}

The gene structures of all identified cucumber WRKY genes were identified by the Gene Structure Display Server (GSDS, http://gsds.cbi.pku.edu.cn/). The cucumber WRKY genes were classified into different groups according to the classification scheme of Arabidopsis WRKY genes and the WRKY domain alignments of CsWRKY and AtWRKY proteins. Alignments of the amino acid sequences of the following were performed using ClustalX with default settings: WRKY domains from cucumber and Arabidopsis (excluding the Cterminal domains of Group I); 61 full-length CsWRKYs; and the Group IIa WRKY domains from Arabidopsis [16], castor bean [64], cucumber, grape [67], tomato [19], pear [63], potato [66], poplar [62], barley [59], rice [17], maize [18], bread wheat [51], Brachypodium [57] and millet [81]. Phylogenetic trees were then constructed based on the alignments using the neighbour-joining (NJ) method of MEGA 5.0. The trees were visualized and optimized via Evolview (http://www.evolgenius.info/ evolview).

\section{Motif composition analysis of CsWRKY proteins}

The motifs within the 61 cucumber WRKY protein sequences were identified using the MEME online program (http://meme.nbcr.net/meme/intro.html) with the following parameters: number of repetitions, any; maximum number of motifs, 10; and optimum width of each motif, between 6 and 300 residues.

\section{Analysis of gene duplication}

The Multiple Collinearity Scan toolkit (MCScanX) was used to examine the gene duplication events, with the default parameters [82]. To explore the syntenic relationships of the WRKY genes obtained from cucumber and other selected species, syntenic analysis maps were constructed using MCScanX.

\section{Regulatory elements in the promoter regions of CsWRKY genes}

The elements in the $1.5 \mathrm{~kb}$ promoter fragments (upstream sequences of the CsWRKY-encoding sequences) of the CsWRKY genes were analysed using the online PlantCARE database (http://bioinformatics.psb.ugent.be/ webtools/plantcare/html/).

\section{Transcriptome analysis of WRKY genes in cucumber}

The expression patterns of the CsWRKYs were analysed based on published RNA-seq data (SRA046916) [69]. Clean tags were remapped to the cucumber genome 
sequence (http://cucurbitgenomics.org/, v3.0) by Biomarker Technologies (Beijing, China), and the FPKM values were recalculated. These analyses were performed on 9 different cucumber tissues: roots, stems, leaves, female flowers, male flowers, ovaries, expanded unfertilized ovaries, expanded fertilized ovaries, and tendrils. Only one biological replication was used for each tissue sample [69]. The genome-wide expression of the CsWRKY genes was shown on a heatmap using MeV (Multiple Experiment Viewer) software, and the expression levels are shown by a colour bar that changes from green to red.

\section{Transcriptome analysis of CsWRKYs in response to abiotic and biotic stresses}

The expression regulation of CsWRKY genes responsive to different stresses was obtained from publicly available transcriptomic data, which were downloaded from Gene Expression Omnibus and analysed to reveal the genomewide differentially expressed genes after treatment with salt (GSE116265) [70] and inoculation with DM (SRP009350) [54] and PM (GSE81234) [71]. Every treatment had three or two biological replicates. The FDR (or $P$ value) and absolute value of $\log 2$ (fold-change) that were published in the original literature were used for the identification of DEGs [54, 70, 71]. Because the gene ID shown was according to the cucumber genome v2.0, we crossreferenced the gene IDs of the CsWRKYs with those of the cucumber genome v3.0. The expression of the CsWRKY genes was then shown by a heatmap using $\mathrm{MeV}$ software.

The seedlings of the 'Chinese long' inbred line 9930, which was obtained from X. Gu Lab of Institute of Vegetables and Flowers, Chinese Academy of Agricultural Sciences, and used for cucumber genome sequencing, were treated at $42^{\circ} \mathrm{C}$, and the leaves of the seedlings were taken at 0,3 and $6 \mathrm{~h}$ after treatment for transcriptome sequencing in Novogene (Beijing, China). Three biological replicates were performed. The transcript abundance of CsWRKY genes was calculated as fragments per kilobase of exon model per million mapped reads (FPKM). The sequencing reads data were submitted to the National Center for Biotechnology Information (NCBI) GEO Sequence Read Archive with accession number of GSE151055.

For the transcriptome analysis of CsWRKYs in response to abiotic and biotic stresses, a threshold of FDR (or $P$ value) $\leq 0.05$ and an absolute value of $\log 2$ (foldchange) $\geq 1$ were used to define DEGs.

\section{Supplementary information}

Supplementary information accompanies this paper at https://doi.org/10. 1186/s12870-020-02625-8.

Additional file 1: Figure S1. The WRKY genes we identified mapped on every chromosome according to the current version of cucumber genome (v3.0)
Additional file 2: Figure S2. The number of WRKY genes mapped on every chromosome according to cucumber genome v1.0 (previous study) and $v 3.0$ (this study).

Additional file 3: Table S1. List of the 61 CSWRKY genes identified in this study.

Additional file 4: Table S2. List of WRKY domains sequence derived from cucumber and Arabidopsis.

Additional file 5: Table S3. List of WRKY domain sequences of WRKY lla proteins.

Additional file 6: Table S4. Analysis of conserved motifs in cucumber WRKY proteins.

Additional file 7: Table S5. Segmentally duplicated CSWRKY gene pairs. Additional file 8: Table S6. Orthologous relationships between cucumber and Arabidopsis/tomato/watermelon/rice/maize.

Additional file 9: Table S7. List FPKM of WRKY genes in cucumber different tissues.

Additional file 10: Table S8. RNA-seq data (FPKM values) of CSWRKY genes under salt/heat treatment/powdery mildew /downy mildew infection.

Additional file 11: Figure S3. Hierarchical clustering of cucumber gene expression profiles under heat treatment. A, Hierarchical cluster of expressed cucumber genes in 9 samples. In the color panels, each transverse line represents a single gene and the color of the line indicates the expression level of the gene relative to the mean center in a specific sample: red, high expression; green, low expression level. B, The hierarchical clustering on the gene expression matrix, using the Pearson correlation coefficient as a proxy of similarity between transcriptomes. $\mathrm{HTOh}=$ heat treatment for $0 \mathrm{~h}$ (hours), HT3h = heat treatment for $3 \mathrm{~h}$, $\mathrm{HT} 6 \mathrm{~h}=$ heat treatment for $6 \mathrm{~h}$

\section{Abbreviations}

TFs: Transcription factors; HMM: Hidden Markov Model; CDS: Coding DNA sequence; MW: Molecular weight; pl: Isoelectric point; aa: amino acid; NJ: Neighbor-joining; R protein: Resistance protein; ORF: Open reading frame; DEGs: Differential expression genes; h: Hours; Si: Silicon; DM: Downy mildew; PM: Powdery mildew

\section{Acknowledgements}

We sincerely thank Dr. Shuai Yin for the checking of the manuscript.

\section{Authors' contributions}

ZR and CC conceived and designed the experiments. XC and JH provided transcriptome data. CC analyzed the data and wrote the manuscript. All authors have read and approved the final manuscript.

\section{Funding}

This work was funded by the National Natural Science Foundation of China (31872950, 31672170 and 31701923), the Shandong "Double Tops" Program (SYL2017YSTD06) and the Taishan Scholar' Foundation of the People's Government of Shandong Province (ts20130932). The funders had no role in study design, data collection and analysis, data interpretation, or in writing of the manuscript.

\section{Availability of data and materials}

The datasets supporting the conclusions of this article are included within the article and its additional files; Cucumber sequences in this article can be found from the CuGenDB (http://cucurbitgenomics.org/); The Arabidopsis thaliana sequences in this article were downloaded from TAIR (https://www. arabidopsis.org/index.jsp). The public transcriptome data are available at Gene Expression Omnibus (GEO) (https://www.ncbi.nlm.nih.gov/geo/). The sequencing reads data of cucumber in response to high temperature stress are deposited at GEO with accession number of GSE151055 (https://www. ncbi.nlm.nih.gov/geo/query/acc.cgi?acc=GSE151055). All plant materials were selected from cucumber provided by the Z. Ren lab, Shandong Agricultural University, Taian, China. 


\section{Ethics approval and consent to participate}

Not applicable.

\section{Consent for publication}

Not applicable.

\section{Competing interests}

The authors declare that they have no competing interest.

\section{Author details}

'State Key Laboratory of Crop Biology, Shandong Collaborative Innovation Center of Fruit \& Vegetable Quality and Efficient Production, Tai'an, People's Republic of China. ${ }^{2}$ Key Laboratory of Biology and Genetic Improvement of Horticultural Crops in Huang-Huai Region, Ministry of Agriculture, College of Horticulture Science and Engineering, Shandong Agricultural University, Tai'an, Shandong 271018, People's Republic of China.

\section{Received: 7 February 2020 Accepted: 26 August 2020} Published online: 25 September 2020

\section{References}

1. Bai Y, Kissoudis C, Yan Z, Visser RGF, van der Linden G. Plant behaviour under combined stress: tomato responses to combined salinity and pathogen stress. Plant J. 2018;93(4):781-93.

2. Garbeva P, Weisskopf L. Airborne medicine: bacterial volatiles and their influence on plant health. New Phytol. 2020;226(1):32-43.

3. Kang H, Zhang M, Zhou S, Guo Q, Chen F, Wu J, Wang W. Overexpression of wheat ubiquitin gene, Ta-Ub2, improves abiotic stress tolerance of Brachypodium distachyon. Plant Sci. 2016;248:102-15.

4. Boyer JS. Plant productivity and environment. Science. 1982;218(4571):4438.

5. Wang G, Zhang S, Ma X, Wang Y, Kong F, Meng Q. A stress-associated NAC transcription factor (SINAC35) from tomato plays a positive role in biotic and abiotic stresses. Physiol Plant. 2016;158(1):45-64.

6. Basso MF, Ferreira PCG, Kobayashi AK, Harmon FG, Nepomuceno AL, Molinari HBC, Grossi-de-Sa MF. MicroRNAs and new biotechnological tools for its modulation and improving stress tolerance in plants. Plant Biotechnol J. 2019;17(8):1482-500.

7. Niklas KJ. Functional adaptation and phenotypic plasticity at the cellular and whole plant level. J Biosci. 2009;34(4):613-20.

8. Wang G, Xu X, Wang H, Liu Q, Yang X, Liao L, Cai G. A tomato transcription factor, SIDREB3 enhances the tolerance to chilling in transgenic tomato. Plant Physiol Biochem. 2019;142:254-62.

9. Cao ZH, Zhang SZ, Wang RK, Zhang RF, Hao YJ. Genome wide analysis of the apple MYB transcription factor family allows the identification of MdoMYB121 gene confering abiotic stress tolerance in plants. PLoS One. 2013;8(7):e69955.

10. Yan $H$, Jia H, Chen X, Hao L, An H, Guo X. The cotton WRKY transcription factor GhWRKY17 functions in drought and salt stress in transgenic Nicotiana benthamiana through ABA signaling and the modulation of reactive oxygen species production. Plant Cell Physiol. 2014;55(12):2060-76.

11. Zhang YL, Zhang CL, Wang GL, Wang YX, Qi CH, You CX, Li YY, Hao YJ. Apple AP2/EREBP transcription factor MdSHINE2 confers drought resistance by regulating wax biosynthesis. Planta. 2019;249(5):1627-43.

12. Le Henanff G, Profizi C, Courteaux B, Rabenoelina F, Gerard C, Clement C, Baillieul F, Cordelier S, Dhondt-Cordelier S. Grapevine NAC1 transcription factor as a convergent node in developmental processes, abiotic stresses, and necrotrophic/biotrophic pathogen tolerance. J Exp Bot. 2013;64(16): 4877-93.

13. Liu B, Ouyang Z, Zhang Y, Li X, Hong Y, Huang L, Liu S, Zhang H, Li D, Song F. Tomato NAC transcription factor SISRN1 positively regulates defense response against biotic stress but negatively regulates abiotic stress response. PLoS One. 2014;9(7):e102067.

14. Riechmann JL, Ratcliffe OJ. A genomic perspective on plant transcription factors. Curr Opin Plant Biol. 2000;3(5):423-34.

15. Ulker B, Somssich IE. WRKY transcription factors: from DNA binding towards biological function. Curr Opin Plant Biol. 2004;7(5):491-8.

16. Dong J, Chen C, Chen Z. Expression profiles of the Arabidopsis WRKY gene superfamily during plant defense response. Plant Mol Biol. 2003;51(1):21-37.

17. Ross CA, Liu Y, Shen QJ. The WRKY gene family in rice (Oryza sativa). J Integr Plant Biol. 2007;49(6):827-42.
18. Zhang T, Tan D, Zhang L, Zhang X, Han Z. Phylogenetic analysis and drought-responsive expression profiles of the WRKY transcription factor family in maize. Agri Gene. 2017;3:99-108.

19. Huang S, Gao Y, Liu J, Peng X, Niu X, Fei Z, Cao S, Liu Y. Genome-wide analysis of WRKY transcription factors in Solanum lycopersicum. Mol Genet Genomics. 2012;287(6):495-513.

20. Rinerson Cl, Rabara RC, Tripathi P, Shen QJ, Rushton PJ. The evolution of WRKY transcription factors. BMC Plant Biol. 2015;15:66.

21. An JP, Zhang XW, You CX, Bi SQ, Wang XF, Hao YJ. MdWRKY40 promotes wounding-induced anthocyanin biosynthesis in association with MdMYB1 and undergoes MdBT2-mediated degradation. New Phytol. 2019;224(1):38095.

22. Xie T, Chen C, Li C, Liu J, Liu C, He Y. Genome-wide investigation of WRKY gene family in pineapple: evolution and expression profiles during development and stress. BMC Genomics. 2018;19(1):490.

23. Wu KL, Guo ZJ, Wang HH, Li J. The WRKY family of transcription factors in rice and Arabidopsis and their origins. DNA Res. 2005;12(1):9-26.

24. Zhang $Y$, Wang $L$. The WRKY transcription factor superfamily: its origin in eukaryotes and expansion in plants. BMC Evol Biol. 2005:5:1.

25. Eulgem T, Rushton PJ, Robatzek S, Somssich IE. The WRKY superfamily of plant transcription factors. Trends Plant Sci. 2000;5(5):199-206.

26. Rushton PJ, Somssich IE, Ringler P, Shen QJ. WRKY transcription factors. Trends Plant Sci. 2010;15(5):247-58.

27. Gu L, Dou L, Guo Y, Wang H, Li L, Wang C, Ma L, Wei H, Yu S. The WRKY transcription factor GhWRKY27 coordinates the senescence regulatory pathway in upland cotton (Gossypium hirsutum L.). BMC Plant Biol. 2019; 19(1):116.

28. Zhang S, Li C, Wang R, Chen Y, Shu S, Huang R, Zhang D, Li J, Xiao S, Yao $\mathrm{N}$, et al. The Arabidopsis Mitochondrial Protease FtSH4 Is Involved in Leaf Senescence via Regulation of WRKY-Dependent Salicylic Acid Accumulation and Signaling. Plant Physiol. 2017;173(4):2294-307.

29. Pesch M, Dartan B, Birkenbihl R, Somssich IE, Hulskamp M. Arabidopsis TTG2 regulates TRY expression through enhancement of activator complextriggered activation. Plant Cell. 2014;26(10):4067-83.

30. Duan S, Wang J, Gao C, Jin C, Li D, Peng D, Du G, Li Y, Chen M. Functional characterization of a heterologously expressed Brassica napus WRKY41-1 transcription factor in regulating anthocyanin biosynthesis in Arabidopsis thaliana. Plant Sci. 2018:268:47-53.

31. Gonzalez A, Brown M, Hatlestad G, Akhavan N, Smith T, Hembd A, Moore J, Montes D, Mosley T, Resendez J, et al. TTG2 controls the developmental regulation of seed coat tannins in Arabidopsis by regulating vacuolar transport steps in the proanthocyanidin pathway. Dev Biol. 2016;419(1):54-63.

32. Chen M, Yan T, Shen Q, Lu X, Pan Q, Huang Y, Tang Y, Fu X, Liu M, Jiang W, et al. GLANDULAR TRICHOME-SPECIFIC WRKY 1 promotes artemisinin biosynthesis in Artemisia annua. New Phytol. 2017;214(1):304-16.

33. Yu Y, Liu Z, Wang L, Kim SG, Seo PJ, Qiao M, Wang N, Li S, Cao X, Park CM, et al. WRKY71 accelerates flowering via the direct activation of FLOWERING LOCUS T and LEAFY in Arabidopsis thaliana. Plant J. 2016;85(1):96-106.

34. Zhang $L$, Chen L, Yu D. Transcription Factor WRKY75 Interacts with DELLA Proteins to Affect Flowering. Plant Physiol. 2018;176(1):790-803.

35. Johnson CS, Kolevski B, Smyth DR. TRANSPARENT TESTA GLABRA2, a trichome and seed coat development gene of Arabidopsis, encodes a WRKY transcription factor. Plant Cell. 2002;14(6):1359-75.

36. Jiang W, YU D. Arabidopsis WRKY2 transcription factor mediates seed germination and postgermination arrest of development by abscisic acid. BMC Plant Biol. 2009;9:96.

37. Ding ZJ, Yan JY, Li GX, Wu ZC, Zhang SQ, Zheng SJ. WRKY41 controls Arabidopsis seed dormancy via direct regulation of $A B / 3$ transcript levels not downstream of ABA. Plant J. 2014:79(5):810-23.

38. Jiang Y, Deyholos MK. Comprehensive transcriptional profiling of $\mathrm{NaCl}$ stressed Arabidopsis roots reveals novel classes of responsive genes. BMC Plant Biol. 2006;6:25

39. Chen YF, Li LQ, Xu Q, Kong YH, Wang H, Wu WH. The WRKY 6 transcription factor modulates PHOSPHATE1 expression in response to low Pi stress in Arabidopsis. Plant Cell. 2009;21(11):3554-66.

40. Kim KC, Lai Z, Fan B, Chen Z. Arabidopsis WRKY38 and WRKY62 transcription factors interact with histone deacetylase 19 in basal defense. Plant Cell. 2008;20(9):2357-71.

41. Lai Z, Vinod K, Zheng Z, Fan B, Chen Z. Roles of Arabidopsis WRKY3 and WRKY4 transcription factors in plant responses to pathogens. BMC Plant Biol. 2008;8(1):68. 
42. Chen X, Liu J, Lin G, Wang A, Wang Z, Lu G. Overexpression of AtWRKY28 and AtWRKY75 in Arabidopsis enhances resistance to oxalic acid and Sclerotinia sclerotiorum. Plant Cell Rep. 2013;32(10):1589-99.

43. Pandey SP, Roccaro M, Schon M, Logemann E, Somssich IE. Transcriptional reprogramming regulated by WRKY18 and WRKY40 facilitates powdery mildew infection of Arabidopsis. Plant J. 2010;64(6):912-23.

44. Jammes F, Lecomte P. de Almeida-Engler J, Bitton F, Martin-Magniette ML, Renou JP, Abad P, Favery B. Genome-wide expression profiling of the host response to root-knot nematode infection in Arabidopsis. Plant J. 2005;44(3): 447-58.

45. Grunewald W, Karimi M, Wieczorek K, Van de Cappelle E, Wischnitzki E, Grundler F, Inze D, Beeckman T, Gheysen G. A role for AtWRKY23 in feeding site establishment of plant-parasitic nematodes. Plant Physiol. 2008;148(1): 358-68

46. Bai Y, Sunarti S, Kissoudis C, Visser RGF, van der Linden CG. The Role of Tomato WRKY Genes in Plant Responses to Combined Abiotic and Biotic Stresses. Front Plant Sci. 2018;9:801.

47. He Y, Mao S, Gao Y, Zhu L, Wu D, Cui Y, Li J, Qian W. Genome-Wide Identification and Expression Analysis of WRKY Transcription Factors under Multiple Stresses in Brassica napus. PLoS One. 2016;11(6):e0157558.

48. Yu Y, Wang N, Hu R, Xiang F. Genome-wide identification of soybean WRKY transcription factors in response to salt stress. Springerplus. 2016;5(1):920.

49. Jimmy JL, Babu S. Role of OsWRKY transcription factors in rice disease resistance. Trop Plant Pathol. 2015;40(6):355-61.

50. Chen L, Zhao Y, Xu S, Zhang Z, Xu Y, Zhang J, Chong K. OsMADS57 together with OsTB1 coordinates transcription of its target OsWRKY94 and D14 to switch its organogenesis to defense for cold adaptation in rice. New Phytol. 2018;218(1):219-31.

51. Okay S, Derelli E, Unver T. Transcriptome-wide identification of bread wheat WRKY transcription factors in response to drought stress. Mol Genet Genomics. 2014;289(5):765-81.

52. Huang S, Li R, Zhang Z, Li L, Gu X, Fan W, Lucas WJ, Wang X, Xie B, Ni P, et al. The genome of the cucumber, Cucumis sativus L. Nat Genet. 2009;41(12): 1275-81.

53. Luan Q, Chen C, Liu M, Li Q, Wang L, Ren Z. CsWRKY50 mediates defense responses to Pseudoperonospora cubensis infection in Cucumis sativus. Plant Sci. 2019;279:59-69.

54. Adhikari BN, Savory EA, Vaillancourt B, Childs KL, Hamilton JP, Day B, Buell CR. Expression profiling of Cucumis sativus in response to infection by Pseudoperonospora cubensis. PLoS One. 2012;7(4):e34954.

55. Ling J, Jiang W, Zhang Y, Yu H, Mao Z, Gu X, Huang S, Xie B. Genome-wide analysis of WRKY gene family in Cucumis sativus. BMC Genomics. 2011;12: 471.

56. Li Q, Li H, Huang W, Xu Y, Zhou Q, Wang S, Ruan J, Huang S, Zhang Z. A chromosome-scale genome assembly of cucumber (Cucumis sativus L.). Gigascience. 2019;8(6).

57. Tripathi P, Rabara RC, Langum TJ, Boken AK, Rushton DL, Boomsma DD, Rinerson Cl, Rabara J, Reese RN, Chen X, et al. The WRKY transcription factor family in Brachypodium distachyon. BMC Genomics. 2012;13:270.

58. Ding $M$, Chen J, Jiang $Y$, Lin L, Cao $Y$, Wang $M$, Zhang $Y$, Rong J, Ye W. Genome-wide investigation and transcriptome analysis of the WRKY gene family in Gossypium. Mol Genet Genomics. 2014;290(1):151-71.

59. Mangelsen E, Kilian J, Berendzen KW, Kolukisaoglu üH, Harter K, Jansson C, Wanke D. Phylogenetic and comparative gene expression analysis of barley (Hordeum vulgare) WRKY transcription factor family reveals putatively retained functions between monocots and dicots. BMC Genomics. 2008;9(1): 194.

60. Wei $Y$, Haitao S, Zhiqiang X, Weiwei T, Zehong D, Yan Y, Wenquan W, Wei $H$, Kaimian L. Genome-Wide Identification and Expression Analysis of the WRKY Gene Family in Cassava. Front Plant Sci. 2016;7.

61. Raja K, Sriram V, Backiyarani S, Uma S, Saraswathi MS, Mayilvaganan M. Evolutionary Expansion of WRKY Gene Family in Banana and Its Expression Profile during the Infection of Root Lesion Nematode, Pratylenchus coffeae. Plos One. 2016;11(9):e0162013.

62. He H, Dong Q, Shao Y, Jiang H, Xiang Y. Genome-wide survey and characterization of the WRKY gene family in Populus trichocarpa. Plant Cell Rep. 2012;31(7):1199-217

63. Huang X, Li K, Xu X, Yao Z, Jin C, Zhang S. Genome-wide analysis of WRKY transcription factors in white pear (Pyrus bretschneideri) reveals evolution and patterns under drought stress. BMC Genomics. 2015;16(1):1104
64. Li HL, Zhang LB, Guo D, Li CZ, Peng SQ. Identification and expression profiles of the WRKY transcription factor family in Ricinus communis. Gene. 2012;503(2):248-53.

65. Li D, Liu P, Yu J, Wang L, Dossa K, Zhang Y, Zhou R, Wei X, Zhang X. Genome-wide analysis of WRKY gene family in the sesame genome and identification of the WRKY genes involved in responses to abiotic stresses. BMC Plant Biol. 2017;17(1):152.

66. Liu QN, Liu Y, Xin Z-Z, Zhang D-Z, Ge B-M, Yang R-P, Wang Z-F, Yang L, Tang B-P, Zhou C-L. Genome-wide identification and characterization of the WRKY gene family in potato (Solanum tuberosum). Biochem Syst Ecol. 2017; 71:212-8.

67. Zhang Y, Feng JC. Identification and Characterization of the Grape WRKY Family. Biomed Res Int. 2014;2014:787680.

68. Hu R, Qi G, Kong Y, Kong D, Gao Q, Zhou G. Comprehensive analysis of NAC domain transcription factor gene family in Populus trichocarpa. BMC Plant Biol. 2010;10:145

69. Li Z, Zhang Z, Yan P, Huang S, Lin K. RNA-Seq improves annotation of protein-coding genes in the cucumber genome. BMC Genomics. 2011;12(1): 540.

70. Zhu Y, Yin J, Liang Y, Liu J, Jia J. Transcriptomic dynamics provide an insight into the mechanism for silicon-mediated alleviation of salt stress in cucumber plants. Ecotoxicol Environ Saf. 2019:174:245-54.

71. Xu Q, Xu X, Shi Y, Qi X, Chen X. Elucidation of the molecular responses of a cucumber segment substitution line carrying Pm5.1 and its recurrent parent triggered by powdery mildew by comparative transcriptome profiling. BMC Genomics. 2017;18(1):21.

72. Debona D, Rodrigues FA, Datnoff LE. Silicon's Role in Abiotic and Biotic Plant Stresses. Annu Rev Phytopathol. 2017;55:85-107.

73. Brand LH, Fischer NM, Harter K, Kohlbacher O, Wanke D. Elucidating the evolutionary conserved DNA-binding specificities of WRKY transcription factors by molecular dynamics and in vitro binding assays. Nucleic Acids Res. 2013:41(21):9764-78.

74. Wei KF, Chen J, Chen YF, Wu LJ, Xie DX. Molecular phylogenetic and expression analysis of the complete WRKY transcription factor family in maize. DNA Res. 2012;19(2):153-64.

75. Xu Z, Sun L, Zhou Y, Yang W, Cheng T, Wang J, Zhang Q. Identification and expression analysis of the SQUAMOSA promoter-binding protein (SBP)-box gene family in Prunus mume. Mol Genet Genomics. 2015;290(5):1701-15.

76. Grunewald W, Smet ID, Lewis DR, Löfke C, Beeckman T. Transcription factor WRKY23 assists auxin distribution patterns during Arabidopsis root development through local control on flavonol biosynthesis. Proc Natl Acad Sci U S A. 2012:109(5):1554-9.

77. Stetter MG, Benz M, Ludewig U. Increased root hair density by loss of WRKY6 in Arabidopsis thaliana. Peerj. 2017:5(5):e2891.

78. Ramamoorthy R, Jiang SY, Kumar N, Venkatesh PN, Ramachandran S. A comprehensive transcriptional profiling of the WRKY gene family in rice under various abiotic and phytohormone treatments. Plant Cell Physiol. 2008:49(6):865-79.

79. Scarpeci TE, Zanor MI, Mueller-Roeber B, Valle EM. Overexpression of AtWRKY30 enhances abiotic stress tolerance during early growth stages in Arabidopsis thaliana. Plant Mol Biol. 2013;83(3):265-77.

80. Liu Q, Li X, Yan S, Yu T, Yang J, Dong J, Zhang S, Zhao J, Yang T, Mao X. OsWRKY67 positively regulates blast and bacteria blight resistance by direct activation of $P R$ genes in rice. BMC Plant Biol. 2018;18(1).

81. Muthamilarasan M, Bonthala VS, Khandelwal R, Jaishankar J, Shweta S, Nawaz K, Prasad M. Global analysis of WRKY transcription factor superfamily in Setaria identifies potential candidates involved in abiotic stress signaling. Front Plant Sci. 2015;6:910

82. Wang $Y$, Tang H, DeBarry JD, Tan X, Li J, Wang X, Lee T-h, Jin H, Marler B, Guo H. MCScanX: a toolkit for detection and evolutionary analysis of gene synteny and collinearity. Nucleic Acids Res. 2012;40(7):e49.

\section{Publisher's Note}

Springer Nature remains neutral with regard to jurisdictional claims in published maps and institutional affiliations. 